(C) 2017. This manuscript version is made available under the CC-BY-NC-ND 4.0 license http:// creativecommons.org/licenses/by-nc-nd/4.0/ 


\title{
Topology optimization for functionally graded cellular composites with metamaterials by level sets
}

\author{
Hao Li ${ }^{\text {a,b }}$, Zhen Luo ${ }^{\text {b,* }}$, Liang Gao ${ }^{\text {a,*** }}$, Paul Walker ${ }^{\text {b }}$ \\ a State Key Lab of Digital Manufacturing Equipment and Technology \\ Huazhong University of Science and Technology, 1037 Luoyu Road, Wuhan, Hubei 430074, China \\ ${ }^{b}$ School of Electrical, Mechanical and Mechatronic Systems \\ University of Technology, Sydney, 15 Broadway, Ultimo, NSW 2007 Australia \\ "Corresponding author in submission: Phone: +61 29514 2994; E-mail: zhen.luo@uts.edu.au (Dr Z. Luo) \\ **Corresponding author in publication: Phone: +86278755 9419; E-mail: gaoliang@mail.hust.edu.cn (Prof L. Gao)
}

\begin{abstract}
The application of auxetic composites in practice often relies on a compromise between properties as auxetics are mostly too porous (not dense enough or not stiff enough) to bear structural loads. Hence, the focus of this paper is topological design optimization of new functionally graded cellular composites with auxetics using a level set method. Firstly, a new hierarchical multi-scale formulation is developed to account for both the auxetic behaviour of the microstructure and the stiffness of the macrostructure. The composite, comprising multiple layers of periodic microstructures, is tailored to have functionally graded properties for stiffness and auxetic behaviours, subject to volumetric gradient constraints. Secondly, the microstructures underpinning composite layers are topologically designed under the consideration of boundary and loading conditions of the macrostructure. Finally, a level set method is applied to evolve the shape and topology of the microstructure for each layer, with the numerical homogenization method to evaluate the effective properties of the microstructures. Several numerical examples are used to demonstrate the effectiveness of the proposed method. It can be seen that such composites systematically gear together the features of the functionally graded materials, cellular composites, and metamaterials towards a new kind of man-made composites.
\end{abstract}

Keywords: Topology optimization; Level set method; Functionally graded materials (FGMs); Cellular composites; Auxetic metamaterials. 


\section{Introduction}

Metamaterials are artificially designed composites engineered to have unusual properties that are difficult to find in nature $[1,2]$. Auxetic metamaterials are a special class of elastic materials that exhibit negative Poisson's ratio (NPR) [3, 4]. In contrast to most conventional materials with positive Poisson's ratios, auxetic materials contract in transverse directions when they are compressed uniaxially. In traditional auxetics, the phenomena of negative Poisson's ratio is associated with specific mechanisms of microstructural deformation which allows rotating effects, e.g. re-entrant, chiral and rotating-units structures [5]. Auxetic metamaterials can find a wide range of applications, e.g. energy absorption, antiimpact, indent resistance, thermal isolation, fracture toughness, acoustic and vibration dampeners, biomedical applications $[6,7]$. However, one of the main limitations of most current NPR composites [8, 9] in practice lies in their lower stiffness under in-plane compression.

The concept of multifunctional cellular composites has grown rapidly in importance in engineering [10, 11]. These porous materials are always characterized with lightweight but high performance. Moreover, cellular composites are flexible in tailoring specific effective properties by modifying their microscopic configurations rather than the constituent materials [12]. Particularly, periodic cellular composites consist of a number of identical microstructures configured in the design space. Hence, the layout of material distribution inside a microstructure provides potential to create cellular composites with multiple functions by using advanced design techniques, such as topology optimization [13, 14]. For instance, with the numerical homogenization method $[13,14]$ to predict the effective properties of a microstructure, topology optimization has been used to design NPR microstructures fashioned from conventional materials, e.g. [1416]. Unfortunately, it can be found that the above studies are mainly focused on the property of NPRs, and the property of the macro structure is seldom included in the design.

Functionally graded materials (FGM) are recognized as a kind of inhomogeneous materials, engineered to have gradient properties by progressively changing the compositions or microstructures over the volume. Studies [17-19] reveal that the biological structures (e.g. bamboo, shell, tooth, bone, etc.) change their mechanical properties layer by layer by varying the constituent materials in order to adapt to environmental stimuli, which can be regarded as the origin of the concept of FGM. This implies that the FGMs can be 
adapted to loading and boundary conditions defined by their service environments in order to improve the mechanical performance, e.g. stiffness, strength and toughness. Inspired by the natural FGMs, engineers start to generate materials with graded properties and multiple functionality for industrial applications, e.g. aerospace and vehicle engineering [20-22]. Hence, we can see that the key concept of FGMs is the incorporation of the multiple functions of different material compositions or microstructures. In practice, the advanced manufacturing in engineering, such as the current additive manufacturing (AM) techniques, will facilitate the application of the man-made FGMs. However, how to achieve FGM properties through the change of material constituents or microstructures over volume is always challenging.

Recently, there is a tendency in implementing man-made FGMs with microstructures by using the topology optimization method [23-26]. In these papers, the numerical homogenization method has been widely used to evaluate the effective FGM properties, when the material properties are subject to small variations [23, 24]. The connectivity issue between adjacent microstructures along the gradient direction of the FGMs has also been widely studied. For instance, Zhou and Li [25] proposed three different approaches to maintain the connectivity between adjacent periodic base cells of the FGMs. Radman et al. [26] developed an efficient method to design the FGMs, in which every three base cells were devised simultaneously and a filtering scheme was performed to preserve the connectivity of the FGMs. However, most of the current works only focused on the single scale design of the FGM microstructures. In engineering, the effect of loading and boundary conditions of the macrostructure upon the microstructure [27-32] should also come into the picture in the topological design of FGMs.

Topology optimization has been recognized as an effective computational design tool for a diversity of structural and material applications [33]. It is a numerical iterative procedure that topologically changes the geometry of the structure within a given design domain subject to boundary conditions until the objective function is optimized. So far, several different methods have been developed for topology optimization, such as the homogenization method [34-36], the solid isotropic material with penalization (SIMP) method $[37,38]$, the evolutionary structural optimization (ESO) method [39] and the level set methods (LSM) [4043]. One of the most promising applications of topology optimization is the synthetic design of material microstructures. 
The numerical homogenization method has been widely applied to topologyoptimization for inverse design of various material microstructures [13-16 44-51]. The numerical homogenization method $[13,14]$ is often used to evaluate the effective properties of the microstructures, and topology optimization methods are applied to determine the topologies of the microstructures. Then the composite material is formulated by periodically configuring a number of microstructures under the assumption that the geometrical sizes of the microstructures are much smaller than that of the macrostructure. Many topological design methods have been developed to create microstructures with extreme or expected properties [13-16, 44-51]. However, most of the above designs are based on the material density distribution methods, e.g. [13-15, 44-50].

LSM 440411 have been used as an alternative method for structural shape and topologyoptimization design problems [42,43,52-58]. The key concept of LSMs is to represent the design boundary of a structure as the zero level set of a higher dimensional level set function. Then, the change of the boundary can be mathematically described by the Hamilton-Jacobi partial differential equation (H-J PDE) [40, 41], and the motion of the boundary is driven by solving the H-J PDE using appropriate numerical methods [40-43]. However, the PDE-driven topology optimization has some strict requirements for numerical implementation (e.g. Courant-Friedrichs-Lewy (CFL) condition, re-initializations and boundary velocity extensions) [40-43, 52]. Furthermore, many well-established structural optimization algorithms, such as the optimality criteria (OC) method [37] and the method of moving asymptotes (MMA) [59] are difficult to apply directly. Hence, several alternative LSMs [60-63] have been proposed in order to overcome the numerical issues in the most conventional level set methods. In particular, the parametric level set method (PLSM) $[61,63]$ has demonstrated its effectiveness as a powerful variant of LSMs. With a given set of compactly supported radial basis functions (CSRBFs) [64], the PLSM converts the complicated PDEdriven shape and topology optimization problem into a much easier "size" optimization problem, so as to keep the desired features while avoiding the numerical issues of the standard LSMs.

This paper systematically integrates the key features of FGMs, cellular composites, and metamaterials into a new family of cellular composites with auxetics. The numerical homogenization method is used to evaluate the effective properties of the microstructures and a topology optimization method using the PLSM 
is applied to evolve the shape and topology of the boundary. The macrostructure is assumed to completely comprise a series of layer-wise cellular composites, and each layer is built from identical microstructures, which facilitates the graded properties of multifunctionality over layers. Such topologically optimized composites can exhibit the desired NPR property while supply sufficient stiffness to bear loads. This paper focuses on the two-dimensional (2D) microstructures with orthotropic and isotropic properties.

\section{Parametric level set method}

The level set method can be briefly illustrated in Fig. 1. The key concept of level set-based methods is to implicitly represent the moving boundary of the structure as the zero level set of a higher dimensional level set function $\Phi$ with Lipschitz continuity. Defining a reference domain D which contains all admissible shapes $\Omega$, each region in the reference domain can be described as:

$$
\left\{\begin{array}{llr}
\Phi(\mathbf{x})>0 & \forall \mathbf{x} \in \Omega \backslash \partial \Omega & \text { (Solid) } \\
\Phi(\mathbf{x})=0 & \forall \mathbf{x} \in \partial \Omega \cap \mathrm{D} & \text { (Boundary) } \\
\Phi(\mathbf{x})<0 & \forall \mathbf{x} \in \mathrm{D} \backslash \Omega & \text { (Void) }
\end{array}\right.
$$

where x denote the point coordinates in $\mathrm{D}$, and $\partial \Omega$ denotes the boundary.

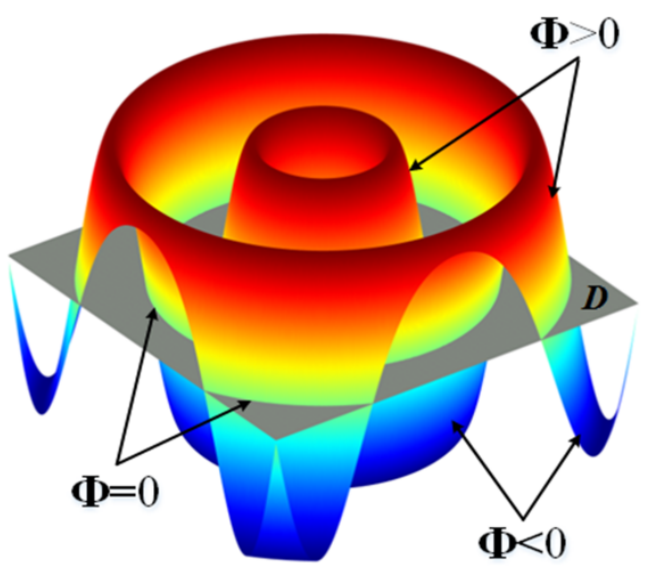

(a)

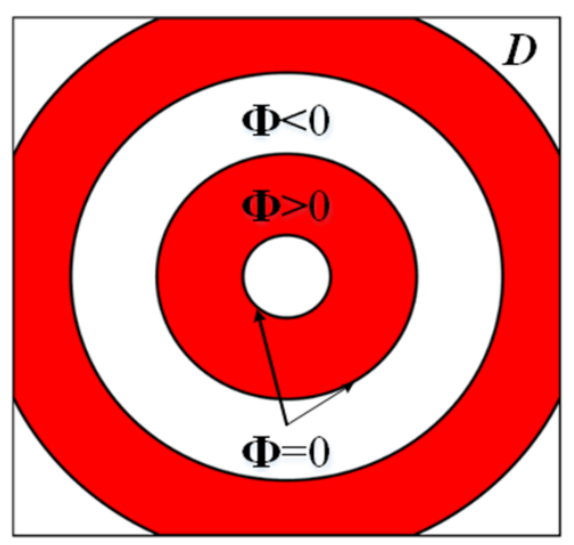

(b)

Figure 1. Level set method: (a) level set function, (b) zero level set and design domain.

Considering a curve $t \mapsto \mathbf{x}(t)$ drawn on the boundary of the evolving domain $\Omega(t)$, the motion of $\Omega(t)$ guiding by the velocity field $\vartheta$ along the outward normal direction of $\partial \Omega$ can be achieved by using the following H-JPDE [40,41]: 


$$
\frac{\partial \Phi(\mathbf{x}, t)}{\partial t}-\vartheta|\nabla \Phi(\mathbf{x}, t)|=0
$$

where $t$ is the pseudo time. The normal velocity field $\vartheta$ can be given by $[42$ 43]:

$$
\vartheta=\left(\frac{d \mathbf{x}(t)}{d t}\right) \cdot \mathbf{n}=\left(\frac{d \mathbf{x}(t)}{d t}\right) \cdot\left(-\frac{\nabla \Phi(\mathbf{x}, t)}{|\nabla \Phi(\mathbf{x}, t)|}\right)
$$

where $\boldsymbol{n}$ is the unit normal vector pointing outward to the moving boundary owing to the sign convention of the leyel set function in this study.

In this paper, the PLSM $[61,63]$ is used to enable shape and topology changes of the design boundary, and the CSRBF [64] is applied to approximate the level set function. Assuming a given set of CSRBFs $\boldsymbol{\varphi ( x )}$ that are positioned statically in the design space, we have

$$
\Phi(\mathbf{x}, t)=\boldsymbol{\varphi}(\mathbf{x}) \cdot \boldsymbol{\alpha}(t)=\sum_{i=1}^{N} \varphi_{i}(\mathbf{x}) \cdot \alpha_{i}(t)
$$

where $N$ is the total number of fixed knots in the design domain, $\alpha_{i}(t)$ is the expansion coefficient of the interpolation with respect to the $i^{\text {th }}$ knot, and $\varphi_{i}(\mathbf{x})$ denotes the CSRBF of the $i^{\text {th }}$ knot which is stated as:

$$
\varphi_{i}(\mathbf{x})=\left(1-r_{i}(\mathbf{x})\right)_{+}^{4}\left(4 r_{i}(\mathbf{x})+1\right) \quad(i=1,2, \ldots, N)
$$

where $r_{i}(\mathbf{x})=\left\|\mathbf{x}-\mathbf{x}_{i}\right\| / r d$ with $r d$ being the radius of the support domain of the knot $\mathbf{x}_{i}$, $[64]$. $\left\|\mathbf{x}-\mathbf{x}_{i}\right\|$ measures the distance from the current sample knot $\mathbf{x}$ to the knot $\mathbf{x}_{i:}(\cdot)_{+}$denotes a truncated power function [64].

It can be seen that the time and spatial terms associated with the dynamic level set function are decoupled via the interpolation. Substituting Eq. (4) into Eq. (2) transforms the H-J PDE into a new form of an ordinary differential equation (ODE):

$$
\boldsymbol{\varphi}(\mathbf{x}) \frac{d \boldsymbol{\alpha}(t)}{d t}-\vartheta \cdot|\nabla \boldsymbol{\varphi}(\mathbf{x}) \boldsymbol{\alpha}(t)|=0
$$

Then, the normal velocity field can be re-written as

$$
\vartheta=\frac{\boldsymbol{\varphi}(\mathbf{x})}{|\nabla \boldsymbol{\varphi}(\mathbf{x}) \boldsymbol{\alpha}(t)|} \dot{\boldsymbol{\alpha}}(t), \text { where } \dot{\boldsymbol{\alpha}}(t)=\frac{d \boldsymbol{\alpha}(t)}{d t}
$$


Hence, the standard LSM has been converted into a parametric form. It is obvious that the velocity field has been extended to the entire design domain as all the terms in Eq. (7) are evaluated over the whole set of the knots in this domain, rather than only the knots along the front. Therefore, no additional numerical scheme is required to extend the velocity field from the boundary to the entire domain. Furthermore the expansion coefficients $\alpha_{i}$ are the only unknowns to be found Therefore if $\alpha_{i}$ are regarded as a set of generalized size parameters it can be seen that the original shape and topology optimization problem has been changed to a general size optimization problem with $\alpha$ being the design variables [61, 63]. In this way, many more efficient gradient-based structural optimization algorithms [37, 59] can be directly applied.

\section{Numerical homogenization method}

In traditional FGMs, two types of approaches can be applied to predict the local effective properties of the material, namely the Mori-Tanaka method [65] or self-consistent method [66]. These rely upon the local volumes, the properties of matrix and the inclusion phases [25]. However, neither of them is directly applicable for evaluating the material properties of the periodic microstructures. The numerical homogenization method $[13,14]$ has been widely used to predict the effective properties of microstructures with periodicity, when the geometrical sizes of the unit cell (e.g. microstructure) are small enough compared to the bulk material. It is noted that the periodicity of the FGM microstructures is not exactly satisfied at the interfaces between different microstructures along the graded direction. However, as illustrated in [2326], the numerical homogenization method is still applicable for approximating the effective properties of microstructures in the design of the layer-wise cellular composites with FGMs, especially when the property gradient variation between different microstructures is relatively small.

Within the framework of the PLSM [61], the numerical homogenization method [13, 14] is applied to approximately evaluate the effective properties of the microstructures at different layers. For a 2D microstructure, the effective elasticity tensor can be calculated by

$$
D_{i j k l}^{H}\left(\mathbf{u}^{M I}, \Phi^{M I}\right)=\frac{1}{\mid \Omega^{M I}} \int_{\Omega^{M I}}\left(\varepsilon_{p q}^{0(i j)}-\varepsilon_{p q}^{*}\left(u^{M I(i j)}\right)\right) D_{p q r s}\left(\varepsilon_{r s}^{0(k l)}-\varepsilon_{r s}^{*}\left(u^{M I(k l)}\right)\right) H\left(\Phi^{M I}\right) d \Omega^{M I}
$$

where $i, j, k, l=1,2 . D_{p q r s}$ is the elasticity tensor of the solid material. The superscript ' $M I$ ' is used to indicate the microscale quantities, such as $\Omega^{M I}$ is the domain of a microstructure, $\left|\Omega^{M I}\right|$ denotes the area of a 
microstructure, and $\Phi^{M I}$ is the microscale level set function. $\varepsilon_{p q}^{0(i j)}$ denote the applied unit strain fields that include the horizontal unit strain $\varepsilon_{p q}^{0(11)}=(1,0,0)^{T}$, the vertical unit strain $\varepsilon_{p q}^{0(22)}=(0,1,0)^{T}$ and the shear unit strain $\varepsilon_{p q}^{0(12)}=(0,0,1)^{T}$ in $2 \mathrm{D}$ [13 314$]$. The locally varying strains $\varepsilon_{p q}^{*}\left(u^{M I(i j)}\right)$ induced by $\varepsilon_{p q}^{0(i j)}$ can be defined by

$$
\varepsilon_{p q}^{*}\left(u^{M I(i j)}\right)=\frac{1}{2}\left(u_{p, q}^{M I(i j)}+u_{q, p}^{M I(i j)}\right)
$$

Here $u^{M I(i j)}$ is the $\Omega^{M I}$-period displacement field in a microstructure, which can be computed by

$$
\int_{\Omega^{M I}}\left(\varepsilon_{p q}^{0(i j)}-\varepsilon_{p q}^{*}\left(u^{M I(i j)}\right)\right) D_{p q r s} \varepsilon_{r s}^{*}\left(v^{M I(i j)}\right) H\left(\Phi^{M I}\right) d \Omega^{M I}=0, \forall v^{M I(i j)} \in \overline{\mathrm{U}}\left(\Omega^{M I}\right)
$$

where $v^{M I(i j)}$ is the virtual displacement field in a microstructure, belonging to the space $\overline{\mathrm{U}}\left(\Omega^{M I}\right)$ which denotes the set of all the kinematically admissible displacements in $\Omega^{M I}$.

In the above, the Heaviside function $H(\Phi)$ [43], which is a sufficiently smooth characteristic function used to distinguish the solid and void in the level set method, can be defined as follows:

$$
H(\Phi)= \begin{cases}\eta, & \Phi<-\Delta \\ \frac{3(1-\eta)}{4}\left(\frac{\Phi}{\Delta}-\frac{\Phi^{3}}{3 \Delta^{3}}\right)+\frac{1+\eta}{2}, & -\Delta \leq \Phi \leq \Delta \\ 1 . & \Phi>\Delta\end{cases}
$$

where $\eta$ is a small positive number to avoid singularity of the numerical process, and $\Delta$ describes the width for the numerical approximation of $H(\Phi) . \delta(\Phi)$ is the derivative of the Heaviside function:

$$
\delta(\Phi)= \begin{cases}\frac{3(1-\eta)}{4 \Delta}\left(1-\frac{\Phi^{2}}{\Delta^{2}}\right), & |\Phi| \leq \Delta \\ 0 . & |\Phi|>\Delta\end{cases}
$$

\section{Topological design of FGMs with auxetics}

\subsection{Layer-wise FGM design}

Here, a layer-wise design concept is developed in engineering for the optimization of FGM-based auxetics with a number of microstructures or representative unit cells (RUCs). The macrostructural design domain is divided into different layers to enable functionally graded properties and then the RUCs, at all individual layers, are optimized concurrently. The concept of layer-wise design is given in Fig. 2. It is assumed that 
the whole macro domain is completely occupied by the composite layers of microstructures, and the topologies of these microstructures account for the performance of the macroscale FGM structure. In this way, the overall topology of the macrostructure remains unchanged, but the properties of its internal microstructures have been optimized due to the change of their topologies.

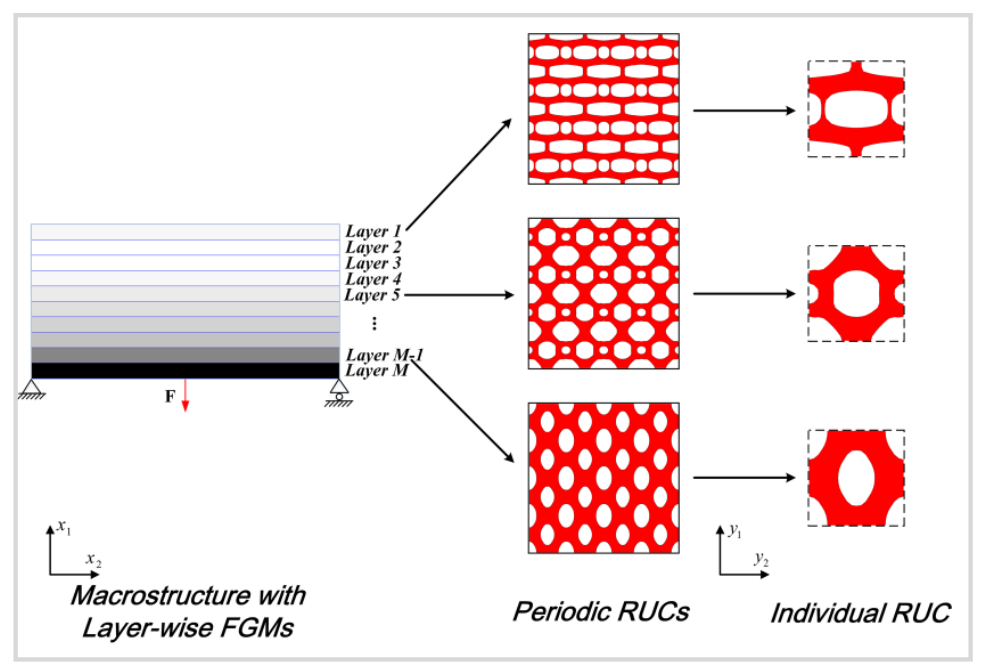

Figure 2. Layer-wise design of FGM-based composite

The layer-wise design concept suits the generation of FGMs in engineering, which possesses several benefits that can be summarized as: (1) The layer-wise design can considerably reduce the computational cost during the optimization due to the fact that only one unique microstructure is designed as a representative for all the identical microstructures at each layer, when comparing to the design with microstructures varying from point to point over the macro design domain; (2) all the microstructures within each individual layer are identical, which will also facilitate manufacturing processes; and (3) the graded properties within the macrostructure can be easily controlled and identified for each layer, which will facilitate the practical applications in engineering.

\subsection{Multi-objective optimization formulation}

This paper focuses on the systematic design of FGMs consisting of layers of auxetic microstructures, to optimize not only the macrostructural stiffness (or minimum mean compliance) but also the microstructural auxetic behavior. In this way, the topological design has multifunctional behaviors, e.g. load bearing and anti-impact capabilities. It is critical to develop the optimization addressing multiple objectives using the PLSM. Here, two different objective functions at the different scales are considered. 
The macro objective of the structural mean compliance can be stated as:

$$
\begin{aligned}
f^{M A}\left(\mathbf{u}^{M A}, \mathbf{u}^{M I}, \Phi^{M I}\right) & =\frac{1}{2} \int_{\Omega^{M A}} \varepsilon_{i j}\left(\mathbf{u}^{M A}\right) D_{i j k l}^{H}\left(\mathbf{u}^{M I}, \Phi^{M I}\right) \varepsilon_{k l}\left(\mathbf{u}^{M A}\right) H\left(\Phi^{M A}\right) d \Omega^{M A} \\
& =\frac{1}{2} \int_{\Omega^{M A}} \varepsilon_{i j}\left(\mathbf{u}^{M A}\right) D_{i j k l}^{H}\left(\mathbf{u}^{M I}, \Phi^{M I}\right) \varepsilon_{k l}\left(\mathbf{u}^{M A}\right) d \Omega^{M A}
\end{aligned}
$$

where $\Omega^{M A}$ represents the macro design domain (the superscript ' $M A$ ' is used to indicate macroscale quantities), $\mathbf{u}^{M A}$ denotes the displacement field in the macro finite element (FE) model, and $\Phi^{M A}$ is the level set function used to represent the topology of the entire macrostructure. $\varepsilon$ denotes the strain field. However, since the topology of the macrostructure is not optimized, the $\Phi^{M A}$ are regarded as a number of positive values that are larger than $\Delta$. The Heaviside function in Eq. (12) can be neglected, as it is always equal to 1 (see Eq. (10)).

Regarding a composite with $M$ different layers (see Fig. 2), the term of macro objective $f^{M A}$ can be reformulated as a summation of the mean compliances $f_{m}^{M A}(m=1,2, \ldots, M)$ from $M$ layers:

$$
f^{M A}\left(\mathbf{u}^{M A}, \mathbf{u}^{M I}, \Phi^{M I}\right)=\sum_{m=1}^{M} f_{m}^{M A}\left(\mathbf{u}_{m}^{M A}, \mathbf{u}_{m}^{M I}, \Phi_{m}^{M I}\right)=\sum_{m=1}^{M} \frac{1}{2} \int_{\Omega_{m}^{M A}} \varepsilon_{i j}\left(\mathbf{u}_{m}^{M A}\right) D_{i j k l}^{H}\left(\mathbf{u}_{m}^{M I}, \Phi_{m}^{M I}\right) \varepsilon_{k l}\left(\mathbf{u}_{m}^{M A}\right) d \Omega_{m}^{M A}
$$

where $m$ denotes that the quantities are defined at layer $m$.

For a material microstructure, one can directly minimize the Poisson's ratios, i.e. $v_{12}=D_{1122}^{H} / D_{1111}^{H}$ or $v_{21}=D_{1122}^{H} / D_{2222}^{H}$, to generate NPR property [15]. Here $v_{12}$ and $v_{21}$ denote the Poisson's ratios along different directions (e.g. $x$ and $y$ directions). For isotropic materials, since $v_{12}=v_{21}$, it doesn't matter either $v_{12}$ or $v_{21}$ is used as an objective function to achieve the minimum of NPR. As a result, the direct formulations are acceptable for the design of isotropic microstructures. However, they are not good candidates for the design of orthotropic microstructures, unless the additional design constraints are used (e.g. the bulk modulus constraint) [15]. This is because when the direct objective function is used, there is a possibility that only one of the two Poisson's ratios $\left(v_{12}\right.$ or $\left.v_{21}\right)$ is minimized, while the other one $\left(v_{21}\right.$ or $v_{12}$ ) may not be optimized. In this case, an orthotropic material will have an obvious auxetic effect in one direction, while a very weak auxetic effect in another direction. Furthermore the identification of the proper bulk modulus constraints for different microstructures is empirical [15] so it may not work well for the current FGM composites that contain a large number of layers (e.g. 40 layers). 
Therefore, to achieve NPR in both the isotropic and orthotropic material microstructures, an alternative micro objective $[14,50]$ at layer $m$ is given as:

$$
f_{m}^{M I}\left(\mathbf{u}_{m}^{M I}, \Phi_{m}^{M I}\right)=D_{1122}^{H}\left(\mathbf{u}_{m}^{M I}, \Phi_{m}^{M I}\right)+D_{2211}^{H}\left(\mathbf{u}_{m}^{M I}, \Phi_{m}^{M I}\right)-2 D_{1212}^{H}\left(\mathbf{u}_{m}^{M I}, \Phi_{m}^{M I}\right)
$$

It is well known that the occurrence of the auxetic behavior is related to the rotating effect of mechanisms, as well as the high shear stiffness within a microstructure. Minimizing the term $D_{1122}^{H}+D_{2211}^{H}$ will enable the generation of mechanism-type layouts that can significantly facilitate the material with NPRs in different directions. At the same time, maximizing $D_{1212}^{H}$ can give a high shear stiffness but prevent the microstructure from forming mechanism-type design. As noted in [14] by Professor Sigmund, the attainable values of $D_{1122}^{H}$ (or $D_{2211}^{H}$ ) and $D_{1212}^{H}$ should be considered simultaneously in devising the auxetic metamaterials. Therefore, the objective function (14) can concurrently minimize the Poisson's ratios $v_{12}$ and $v_{21}$, and is applicable not only to the isotropic but also to the orthotropic materials. Furthermore, considering the objective functions (13) and (14) simultaneously, a multi-objective optimization formulation can automatically determine the effective elasticity tensor as well as the optimal values of $v_{12}$ and $v_{21}$ for each microstructure, in order to minimize the Poisson's ratios along both directions and the mean compliance of the entire structure.

In this paper, the optimization formulation for a layer-wise composite structure is divided into $M$ suboptimization models, in order to achieve the expected gradient properties over different layers. Here, the normalized exponential weighted criterion (NEWC) $[67,68]$ is adopted to handle the multi-objective formulation, because it can be found $[67,68]$ that the NEWC has a good performance to capture the entire Pareto set for optimization problems with both the convex and non-convex Pareto frontiers.

$$
\begin{gathered}
J_{m}^{M A}\left(\mathbf{u}_{m}^{M A}, \mathbf{u}_{m}^{M I}, \Phi_{m}^{M I}\right)=\left(\exp \left(q w_{m, a}\right)-1\right) \exp \left(q\left(\frac{f_{m}^{M A}\left(\mathbf{u}_{m}^{M A}, \mathbf{u}_{m}^{M I}, \Phi_{m}^{M I}\right)-f_{m, \text { min }}^{M A}}{f_{m, \text { max }}^{M A}-f_{m, \text { min }}^{M A}}\right)\right) \\
J_{m}^{M I}\left(\mathbf{u}_{m}^{M I}, \Phi_{m}^{M I}\right)=\left(\exp \left(q w_{m, b}\right)-1\right) \exp \left(q\left(\frac{f_{m}^{M I}\left(\mathbf{u}_{m}^{M I}, \Phi_{m}^{M I}\right)-f_{m, \text { min }}^{M I}}{f_{m, \text { max }}^{M I}-f_{m, \text { min }}^{M I}}\right)\right)
\end{gathered}
$$

where the weight parameters $w_{m, a}$ and $w_{m, b}$ reflect the weighting on the two objectives at the $m^{\text {th }}$ layer, and the summation of $w_{m, a}$ and $w_{m, b}$ is equivalent to $1 . f_{m, \min }^{M A}, f_{m, \max }^{M A}, f_{m, \min }^{M I}$ and $f_{m, \max }^{M I}$ respectively represent the utopia points of the macro and micro objectives, which can be attained by solving the single objective optimization independently (without normalization). $q$ is the exponential constant in the NEWC scheme 
[67], and in this study it is set as $q=2$. In the NEWC, $J_{m}^{M A}$ is the objective function for the mean compliance, and $J_{m}^{M I}$ is the objective function for the NPR property.

The mathematical formulation for the multi-objective topology optimization can be formulated in terms of the summation of two single objective functions given in Eqs. (15) and (16), as follows:

$$
\begin{array}{ll}
\text { Find } & \alpha_{m, n}^{M I}(m=1,2, \ldots, M ; n=1,2, \ldots, N) \\
\text { Min } & J_{m}\left(\mathbf{u}_{m}^{M A}, \mathbf{u}_{m}^{M I}, \Phi_{m}^{M I}\right)=J_{m}^{M A}\left(\mathbf{u}_{m}^{M A}, \mathbf{u}_{m}^{M I}, \Phi_{m}^{M I}\right)+J_{m}^{M I}\left(\mathbf{u}_{m}^{M I}, \Phi_{m}^{M I}\right) \\
\text { S.T. } & G_{m}\left(\Phi_{m}^{M I}\right)=\int_{\Omega_{m}^{M A}} \int_{\Omega_{m}^{M I}} H\left(\Phi_{m}^{M I}\right) d \Omega_{m}^{M I} d \Omega_{m}^{M A} \leq V_{m}^{\max }, \\
& a^{M A}\left(\mathbf{u}^{M A}, \mathbf{v}^{M A}, \mathbf{u}^{M I}, \Phi^{M I}\right)=l^{M A}\left(\mathbf{v}^{M A}\right), \forall \mathbf{v}^{M A} \in \overline{\mathrm{U}}\left(\Omega^{M A}\right), \\
& a^{M I}\left(\mathbf{u}_{m}^{M I}, \mathbf{v}_{m}^{M I}, \Phi_{m}^{M I}\right)=l^{M I}\left(\mathbf{v}_{m}^{M I}, \Phi_{m}^{M I}\right), \forall \mathbf{v}_{m}^{M I} \in \overline{\mathrm{U}}\left(\Omega_{m}^{M I}\right), \\
& \tilde{\alpha}_{m, \min }^{M I} \leq \tilde{\alpha}_{m, n}^{M I} \leq \tilde{\alpha}_{m, \max }^{M I}
\end{array}
$$

where the subscripts $n$ and $m$ indicate the knot $n$ in the level set grid which belongs to the microstructure at the laver $m . N$ is the total number of knots in the level set grid of a microstructure and $M$ is the number of lavers in the macro design space. Concerning with the $m^{\text {th }}$ optimization model (optimization problem at the layer $m), J_{m}$ is the objective function, and $G_{m}$ is the volume constraint with a maximum allowable material usage $V_{m}^{\max }$. u and $\mathbf{v}$ are the real and virtual displacement fields. $\overline{\mathrm{U}}\left(\Omega^{M A}\right)$ denotes the set of all the kinematically admissible displacements in $\Omega^{M A} \overline{\mathrm{U}}\left(\Omega_{m}^{M I}\right)$ represents the set of all the kinematically admissible displacements in $\Omega_{m}^{M I}$ which is the domain of the microstructure at the laver $m . \alpha_{m, n}^{M I}$ denote the expansion coefficients in CSRBF interpolation, which serve as the design variables. $\tilde{\alpha}_{m, \min }^{M I}=0.001$ and $\tilde{\alpha}_{m, \max }^{M I}=1$ are the upper and lower bounds of $\tilde{\alpha}_{m, n \dot{ }}^{M I} \tilde{\alpha}_{m, n}^{M I}$ are regularized design variables, which will be used in the optimization algorithm. The optimization algorithm will be illustrated in Section 4.4.

The bilinear energy form and the linear load form in the macro FE model are given as:

$$
\begin{gathered}
a^{M A}\left(\mathbf{u}^{M A}, \mathbf{v}^{M A}, \mathbf{u}^{M I}, \Phi^{M I}\right)=\int_{\Omega^{M A}} \varepsilon_{i j}\left(\mathbf{u}^{M A}\right) D_{i j k l}^{H}\left(\mathbf{u}^{M I}, \Phi^{M I}\right) \varepsilon_{k l}\left(\mathbf{v}^{M A}\right) d \Omega^{M A} \\
l^{M A}\left(\mathbf{v}^{M A}\right)=\int_{\Omega^{M A}} \mathbf{p} \mathbf{v}^{M A} d \Omega^{M A}+\int_{\partial \Omega^{M A}} \boldsymbol{\tau}^{M A} d \Gamma^{M A}
\end{gathered}
$$

where $\mathbf{p}$ is the body force, and $\boldsymbol{\tau}$ is the traction along the boundary $\Gamma^{M A}$. It should be noticed that the macro displacement fields $\mathbf{u}^{M A}$ and $\mathbf{v}^{M A}$ are defined on $\Omega^{M A}$, and have no periodicity. The bilinear energy form and the linear load form in the micro FE model are stated as:

$$
a^{M I}\left(\mathbf{u}_{m}^{M I}, \mathbf{v}_{m}^{M I}, \Phi_{m}^{M I}\right)=\int_{\Omega_{m}^{M I}} \varepsilon_{p q}^{*}\left(u_{m}^{M I(i j)}\right) D_{p q r s} \varepsilon_{r s}^{*}\left(v_{m}^{M I(i j)}\right) H\left(\Phi_{m}^{M I}\right) d \Omega_{m}^{M I}
$$




$$
l^{M I}\left(\mathbf{v}_{m}^{M I}, \Phi_{m}^{M I}\right)=\int_{\Omega_{m}^{M I}} \varepsilon_{p q}^{0(i j)} D_{p q r s} \varepsilon_{r s}^{*}\left(v_{m}^{M I(i j)}\right) H\left(\Phi_{m}^{M I}\right) d \Omega_{m}^{M I}
$$

where the micro displacement fields $\mathbf{u}_{m}^{M I}$ and $\mathbf{v}_{m}^{M I}$ are defined on $\Omega_{m}^{M I}$. It is noted that $\mathbf{u}_{m}^{M I}$ and $\mathbf{v}_{m}^{M I}$ are under $\Omega_{m}^{M I}$-periodicity $[13,14]$.

The volume constraint $V_{m}^{\max }$ for each sub-optimization problem varies for different layers, so as to enable the gradient properties of different material microstructures over all layers. For instance, the maximum volume fraction of the $m^{\text {th }}$ microstructure of the composite structure can be defined as:

$$
V_{m}^{\max }=V_{1}+\left(V_{M}-V_{1}\right)\left[\frac{(m-1)}{(M-1)}\right]^{\xi}
$$

where $\left[V_{1}, V_{M}\right]$ determines the range of volume constraints. If parameter $\xi=1$ the volume fractions will change linearly, otherwise the volume fractions will vary nonlinearly.

Here, the consideration for choosing the volume fraction gradient is that the material volume is in most cases proportionally related to its structural stiffness. The motivation of this study is to design man-made FGM composites which have multifunctional requirements for both stiffness and auxetic behaviors. Hence, it should be a natural choice to select the volume gradient constraint to allow a well-graded and smooth change of material usage between layers. It is noted that definition of the functional gradient using the above volume constraints is based on numerical experience, and it is difficult to define a general criterion to choose the best functional gradient in practice.

\subsection{Sensitivity Analysis}

In the PLSM, many gradient-based optimization algorithms (e.g. OC and MMA) can be straightforwardly used to update the design variables $\boldsymbol{\alpha}$ given in Eq. (17). Therefore, the first-order derivatives of the objective function and constraint with respect to $\boldsymbol{\alpha}$ are required.

In this section the shape derivative $[69,70]$ is employed to calculate the sensitivity of boundary perturbations with respect to the microscale time variable $t$ by following a similar way to Allaire et al. [42] and Wang et al. [43]. Therefore, the shape derivatives of $D_{i j k l}^{H}\left(\mathbf{u}_{m}^{M I}, \Phi_{m}^{M I}\right), a^{M I}\left(\mathbf{u}_{m}^{M I}, \mathbf{v}_{m}^{M I}, \Phi_{m}^{M I}\right)$ and $l^{M I}\left(\mathbf{v}_{m}^{M I}, \Phi_{m}^{M I}\right)$ can be respectively expressed as: 


$$
\begin{aligned}
\frac{\partial D_{i j k l}^{H}}{\partial t}= & \frac{1}{\left|\Omega_{m}^{M I}\right|} \int_{\Omega_{m}^{M I}}\left(\varepsilon_{p q}^{0(i j)}-\varepsilon_{p q}^{*}\left(u_{m}^{M I(i j)}\right)\right) D_{p q r s}\left(\varepsilon_{r s}^{0(k l)}-\varepsilon_{r s}^{*}\left(u_{m}^{M I}(k l)\right)\right) \vartheta_{m}^{M I}\left|\nabla \Phi_{m}^{M I}\right| \delta\left(\Phi_{m}^{M I}\right) d \Omega_{m}^{M I} \\
& -\frac{2}{\left|\Omega_{m}^{M I}\right|} \int_{\Omega_{m}^{M I}}\left(\varepsilon_{p q}^{*}\left(\dot{u}_{m}^{M I(i j)}\right)\right) D_{p q r s}\left(\varepsilon_{r s}^{0(k l)}-\varepsilon_{r s}^{*}\left(u_{m}^{M I(k l)}\right)\right) H\left(\Phi_{m}^{M I}\right) d \Omega_{m}^{M I} \\
\frac{\partial a^{M I}}{\partial t}= & \int_{\Omega_{m}^{M I}} \varepsilon_{p q}^{*}\left(u_{m}^{M I(i j)}\right) D_{p q r s} \varepsilon_{r s}^{*}\left(v_{m}^{M I(i j)}\right) \vartheta_{m}^{M I}\left|\nabla \Phi_{m}^{M I}\right| \delta\left(\Phi_{m}^{M I}\right) d \Omega_{m}^{M I} \\
& +\int_{\Omega_{m}^{M I}}\left[\varepsilon_{p q}^{*}\left(\dot{u}_{m}^{M I(i j)}\right) D_{p q r s} \varepsilon_{r s}^{*}\left(v_{m}^{M I(i j)}\right)+\varepsilon_{p q}^{*}\left(u_{m}^{M I(i j)}\right) D_{p q r s} \varepsilon_{r s}^{*}\left(\dot{v}_{m}^{M I(i j)}\right)\right] H\left(\Phi_{m}^{M I}\right) d \Omega_{m}^{M I} \\
& \frac{\partial l^{M I}}{\partial t}=\int_{\Omega_{m}^{M I}} \varepsilon_{p q}^{0(i j)} D_{p q r s} \varepsilon_{r s}^{*}\left(v_{m}^{M I(i j)}\right) \vartheta_{m}^{M I}\left|\nabla \Phi_{m}^{M I}\right| \delta\left(\Phi_{m}^{M I}\right) d \Omega_{m}^{M I} \\
& +\int_{\Omega_{m}^{M I}} \varepsilon_{p q}^{0(i j)} D_{p q r s} \varepsilon_{r s}^{*}\left(\dot{v}_{m}^{M I(i j)}\right) H\left(\Phi_{m}^{M I}\right) d \Omega_{m}^{M I}
\end{aligned}
$$

where $\vartheta_{m}^{M I}$ is the microscale normal velocity defined at layer $m$.

In addition, the following conjugate equation can be established:

$$
\int_{\Omega_{m}^{M I}} \varepsilon_{p q}^{*}\left(u_{m}^{M I(i j)}\right) D_{p q r s} \varepsilon_{r s}^{*}\left(\dot{v}_{m}^{M I(i j)}\right) H\left(\Phi_{m}^{M I}\right) d \Omega_{m}^{M I}=\int_{\Omega_{m}^{M I}} \varepsilon_{p q}^{0(i j)} D_{p q r s} \varepsilon_{r s}^{*}\left(\dot{v}_{m}^{M I(i j)}\right) H\left(\Phi_{m}^{M I}\right) d \Omega_{m}^{M I}
$$

Differentiating the microscale equilibrium equation with respect to $t$, yields:

$$
\frac{\partial a^{M I}\left(\mathbf{u}_{m}^{M I}, \mathbf{v}_{m}^{M I}, \Phi_{m}^{M I}\right)}{\partial t}=\frac{\partial l^{M I}\left(\mathbf{v}_{m}^{M I}, \Phi_{m}^{M I}\right)}{\partial t}
$$

Substituting Eqs. (24)-(26) into (27) yields:

$$
\begin{aligned}
& \int_{\Omega_{m}^{M I}}\left(\varepsilon_{p q}^{0(i j)}-\varepsilon_{p q}^{*}\left(u_{m}^{M I(i j)}\right)\right) D_{p q r s} \varepsilon_{r s}^{*}\left(v_{m}^{M I(i j)}\right) \vartheta_{m}^{M I}\left|\nabla \Phi_{m}^{M I}\right| \delta\left(\Phi_{m}^{M I}\right) d \Omega_{m}^{M I} \\
& =\int_{\Omega_{m}^{M I}} \varepsilon_{p q}^{*}\left(\dot{u}_{m}^{M I(i j)}\right) D_{p q r s} \varepsilon_{r s}^{*}\left(v_{m}^{M I(i j)}\right) H\left(\Phi_{m}^{M I}\right) d \Omega_{m}^{M I}
\end{aligned}
$$

Substituting Eq. (28) into (23) and considering the elastic system is self-adjoint [71], the shape derivative of the elasticity tensor is then given as:

where

$$
\begin{gathered}
\frac{\partial D_{i j k l}^{H}}{\partial t}=-\frac{1}{\left|\Omega_{m}^{M I}\right|} \int_{\Omega_{m}^{M I}} \beta\left(\mathbf{u}_{m}^{M I}\right) \vartheta_{m}^{M I}\left|\nabla \Phi_{m}^{M I}\right| \delta\left(\Phi_{m}^{M I}\right) d \Omega_{m}^{M I} \\
\beta\left(\mathbf{u}_{m}^{M I}\right)=\left(\varepsilon_{p q}^{0(i j)}-\varepsilon_{p q}^{*}\left(u_{m}^{M I(i j)}\right)\right) D_{p q r s}\left(\varepsilon_{r s}^{0(k l)}-\varepsilon_{r s}^{*}\left(u_{m}^{M I(k l)}\right)\right)
\end{gathered}
$$

Substituting the microscale normal velocity $\vartheta_{m}^{M I}$ defined in Eq. (7) into Eq. (29), we can get: 


$$
\frac{\partial D_{i j k l}^{H}}{\partial t}=-\sum_{n=1}^{N}\left(\frac{1}{\left|\Omega_{m}^{M I}\right|} \int_{\Omega_{m}^{M I}} \beta\left(\mathbf{u}_{m}^{M I}\right) \varphi_{m, n}^{M I}(\mathbf{x}) \delta\left(\Phi_{m}^{M I}\right) d \Omega_{m}^{M I}\right) \cdot \frac{\partial \alpha_{m, n}^{M I}}{\partial t}
$$

where $\varphi_{m, n}^{M I}$ is the CSRBF of the $n^{\text {th }}$ knot within the microscale design domain of the $m^{\text {th }}$ layer.

On the other hand, the first-order derivative of the effective elasticity tensor with respect to $t$ can be written as following according to the chain rule:

$$
\frac{\partial D_{i j k l}^{H}}{\partial t}=\sum_{n=1}^{N} \frac{\partial D_{i j k l}^{H}}{\partial \alpha_{m, n}^{M I}} \cdot \frac{\partial \alpha_{m, n}^{M I}}{\partial t}
$$

Comparing the corresponding terms in Eqs. (31) and (32), we can easily find the derivatives of the effective elasticity tensor $D_{i j k l}^{H}$ with respect to the design variables, which are given as below:

$$
\frac{\partial D_{i j k l}^{H}}{\partial \alpha_{m, n}^{M I}}=-\frac{1}{\left|\Omega_{m}^{M I}\right|} \int_{\Omega_{m}^{M I}} \beta\left(\mathbf{u}_{m}^{M I}\right) \varphi_{m, n}^{M I}(\mathbf{x}) \delta\left(\Phi_{m}^{M I}\right) d \Omega_{m}^{M I}
$$

The derivatives of $J_{m}^{M A}$ with respect to the design variables $\boldsymbol{\alpha}$ are derived according to the chain rule as:

$$
\frac{\partial J_{m}^{M A}}{\partial \alpha_{m, n}^{M I}}=\left(\exp \left(q w_{m, a}\right)-1\right) \exp \left(q\left(\frac{f_{m}^{M A}-f_{m, \min }^{M A}}{f_{m, \text { max }}^{M A}-f_{m, \min }^{M A}}\right)\right)\left(\frac{q}{f_{m, \text { max }}^{M A}-f_{m, \min }^{M A}} \cdot \frac{\partial f_{m}^{M A}}{\partial \alpha_{m, n}^{M I}}\right)=W_{m, a} \cdot \frac{\partial f_{m}^{M A}}{\partial \alpha_{m, n}^{M I}}
$$

and $W_{m, a}$ denotes the constant term related to the derivatives of the first objective function at the layer $m$, which can be regarded as a coefficient of $\partial f_{m}^{M A} / \partial \alpha_{m, n}^{M I}$.

The derivatives of $f^{M A}\left(\mathbf{u}^{M A}, \mathbf{u}^{M I}, \Phi^{M I}\right), a^{M A}\left(\mathbf{u}^{M A}, \mathbf{v}^{M A}, \mathbf{u}^{M I}, \Phi^{M I}\right)$ and $l^{M A}\left(\mathbf{v}^{M A}\right)$ with respect to the microscale time variable $t$ are given as:

$$
\begin{gathered}
\frac{\partial f^{M A}}{\partial t}=\int_{\Omega^{M A}}\left[\varepsilon_{i j}\left(\dot{\mathbf{u}}^{M A}\right) D_{i j k l}^{H} \varepsilon_{k l}\left(\mathbf{u}^{M A}\right)+\frac{1}{2} \varepsilon_{i j}\left(\mathbf{u}^{M A}\right) \frac{\partial D_{i j k l}^{H}}{\partial t} \varepsilon_{k l}\left(\mathbf{u}^{M A}\right)\right] d \Omega^{M A} \\
\frac{\partial a^{M A}}{\partial t}=\int_{\Omega^{M A}}\left[\varepsilon_{i j}\left(\dot{\mathbf{u}}^{M A}\right) D_{i j k l}^{H} \varepsilon_{k l}\left(\mathbf{v}^{M A}\right)+\varepsilon_{i j}\left(\mathbf{u}^{M A}\right) D_{i j k l}^{H} \varepsilon_{k l}\left(\dot{\mathbf{v}}^{M A}\right)+\varepsilon_{i j}\left(\mathbf{u}^{M A}\right) \frac{\partial D_{i j k l}^{H}}{\partial t} \varepsilon_{k l}\left(\mathbf{v}^{M A}\right)\right] d \Omega^{M A} \\
\frac{\partial l^{M I}}{\partial t}=\int_{\Omega^{M A}} \mathbf{p}^{M A} d \Omega^{M A}+\int_{\partial \Omega^{M A}} \boldsymbol{\tau} \dot{\mathbf{v}}^{M A} d \Gamma^{M A}
\end{gathered}
$$

A conjugate equation can be given as:

$$
\int_{\Omega^{M A}} \varepsilon_{i j}\left(\mathbf{u}^{M A}\right) D_{i j k l}^{H} \varepsilon_{k l}\left(\dot{\mathbf{v}}^{M A}\right) d \Omega^{M A}=\int_{\Omega^{M A}} \mathbf{p} \dot{\mathbf{v}}^{M A} d \Omega^{M A}+\int_{\partial \Omega^{M A}} \boldsymbol{\tau} \dot{\mathbf{v}}^{M A} d \Gamma^{M A}
$$


Differentiating the macroscale equilibrium equation with respect to $t$ :

$$
\frac{\partial a^{M A}\left(\mathbf{u}^{M A}, \mathbf{v}^{M A}, \mathbf{u}^{M I}, \Phi^{M I}\right)}{\partial t}=\frac{\partial l^{M A}\left(\mathbf{v}^{M A}\right)}{\partial t}
$$

Substituting Eqs. (36)-(38) into (39) yields:

$$
\int_{\Omega^{M A}} \varepsilon_{i j}\left(\dot{\mathbf{u}}^{M A}\right) D_{i j k l}^{H} \varepsilon_{k l}\left(\mathbf{v}^{M A}\right) d \Omega^{M A}=-\int_{\Omega^{M A}} \varepsilon_{i j}\left(\mathbf{u}^{M A}\right) \frac{\partial D_{i j k l}^{H}}{\partial t} \varepsilon_{k l}\left(\mathbf{v}^{M A}\right) d \Omega^{M A}
$$

Since that the mean compliance optimization problem is self-adjoint $[42,43,69]$, we can have:

$$
\int_{\Omega^{M A}} \varepsilon_{i j}\left(\dot{\mathbf{u}}^{M A}\right) D_{i j k l}^{H} \varepsilon_{k l}\left(\mathbf{u}^{M A}\right) d \Omega^{M A}=-\int_{\Omega^{M A}} \varepsilon_{i j}\left(\mathbf{u}^{M A}\right) \frac{\partial D_{i j k l}^{H}}{\partial t} \varepsilon_{k l}\left(\mathbf{u}^{M A}\right) d \Omega^{M A}
$$

Substituting Eq. (41) into Eq. (35) yields:

$$
\frac{\partial f^{M A}}{\partial t}=-\frac{1}{2} \int_{\Omega^{M A}} \varepsilon_{i j}\left(\mathbf{u}^{M A}\right) \frac{\partial D_{i j k l}^{H}\left(\mathbf{u}^{M I}, \Phi^{M I}\right)}{\partial t} \varepsilon_{k l}\left(\mathbf{u}^{M A}\right) d \Omega^{M A}
$$

Therefore, the derivative of structural mean compliance at layer $m$ with respect to $t$ is given as:

$$
\frac{\partial f_{m}^{M A}}{\partial t}=-\frac{1}{2} \int_{\Omega_{m}^{M A}} \varepsilon_{i j}\left(\mathbf{u}_{m}^{M A}\right) \frac{\partial D_{i j k l}^{H}\left(\mathbf{u}_{m}^{M I}, \Phi_{m}^{M I}\right)}{\partial t} \varepsilon_{k l}\left(\mathbf{u}_{m}^{M A}\right) d \Omega_{m}^{M A}
$$

Substituting Eq. (32) into Eq. (43) yields:

$$
\frac{\partial f_{m}^{M A}}{\partial t}=-\frac{1}{2} \int_{\Omega_{m}^{M A}} \varepsilon_{i j}\left(\mathbf{u}_{m}^{M A}\right)\left[\sum_{n=1}^{N} \frac{\partial D_{i j k l}^{H}\left(\mathbf{u}_{m}^{M I}, \Phi_{m}^{M I}\right)}{\partial \alpha_{m, n}^{M I}} \cdot \frac{\partial \alpha_{m, n}^{M I}}{\partial t}\right] \varepsilon_{k l}\left(\mathbf{u}_{m}^{M A}\right) d \Omega_{m}^{M A}
$$

On the other hand, the derivative of the objective $f_{m}^{M A}$ with respect to the time variable $t$ can be derived according to the chain rule:

$$
\frac{\partial f_{m}^{M A}}{\partial t}=\sum_{n=1}^{N} \frac{\partial f_{m}^{M A}}{\partial \alpha_{m, n}^{M I}} \cdot \frac{\partial \alpha_{m, n}^{M I}}{\partial t}
$$

Comparing Eq. (44) with Eq. (45), we have: 


$$
\frac{\partial f_{m}^{M A}}{\partial \alpha_{m, n}^{M I}}=-\frac{1}{2} \int_{\Omega_{m}^{M A}} \varepsilon_{i j}\left(\mathbf{u}_{m}^{M A}\right)\left[\frac{\partial D_{i j k l}^{H}\left(\mathbf{u}_{m}^{M I}, \Phi_{m}^{M I}\right)}{\partial \alpha_{m, n}^{M I}}\right] \varepsilon_{k l}\left(\mathbf{u}_{m}^{M A}\right) d \Omega_{m}^{M A}
$$

Similarly, the derivative of the objective function at microscale defined in Eq. (16) can be written as:

$$
\frac{\partial J_{m}^{M I}}{\partial \alpha_{m, n}^{M I}}=\left(\exp \left(q w_{m, b}\right)-1\right) \exp \left(q\left(\frac{f_{m}^{M I}-f_{m, \text { min }}^{M I}}{f_{m, \text { max }}^{M I}-f_{m, \text { min }}^{M I}}\right)\right)\left(\frac{q}{f_{m, \max }^{M I}-f_{m, \min }^{M I}} \cdot \frac{\partial f_{m}^{M I}}{\partial \alpha_{m, n}^{M I}}\right)=W_{m, b} \cdot \frac{\partial f_{m}^{M I}}{\partial \alpha_{m, n}^{M I}}
$$

where $W_{m, b}$ is also defined as a constant to represent all the terms before $\partial f_{m}^{M I} / \partial \alpha_{m, n}^{M I}$, and the derivative of $f_{m}^{M I}$ associated with $\alpha_{m, n}^{M I}$ can be derived as:

$$
\frac{\partial f_{m}^{M I}}{\partial \alpha_{m, n}^{M I}}=\frac{\partial D_{1122}^{H}\left(\mathbf{u}_{m}^{M I}, \Phi_{m}^{M I}\right)}{\partial \alpha_{m, n}^{M I}}+\frac{\partial D_{2211}^{H}\left(\mathbf{u}_{m}^{M I}, \Phi_{m}^{M I}\right)}{\partial \alpha_{m, n}^{M I}}-2 \cdot \frac{\partial D_{1212}^{H}\left(\mathbf{u}_{m}^{M I}, \Phi_{m}^{M I}\right)}{\partial \alpha_{m, n}^{M I}}
$$

Based on Eq. (33), the derivatives defined in Eqs. (46) and (48) can be calculated easily. Then, the derivatives (34) and (47) can be respectively evaluated by virtue of the derivatives (46) and (48). Eventually, with Eqs. (34) and (47), the sensitivity of the aggregated objective function for the multiobjective optimization in Eq. (17) can be obtained as:

$$
\begin{aligned}
\frac{\partial J_{m}}{\partial \alpha_{m, n}^{M I}}= & -\frac{1}{2} W_{m, a} \cdot \int_{\Omega_{m}^{M A}} \varepsilon_{i j}\left(\mathbf{u}_{m}^{M A}\right)\left[\frac{\partial D_{i j k l}^{H}\left(\mathbf{u}_{m}^{M I}, \Phi_{m}^{M I}\right)}{\partial \alpha_{m, n}^{M I}}\right] \varepsilon_{k l}\left(\mathbf{u}_{m}^{M A}\right) d \Omega_{m}^{M A} \\
& +W_{m, b} \cdot\left(\frac{\partial D_{1122}^{H}\left(\mathbf{u}_{m}^{M I}, \Phi_{m}^{M I}\right)}{\partial \alpha_{m, n}^{M I}}+\frac{\partial D_{2211}^{H}\left(\mathbf{u}_{m}^{M I}, \Phi_{m}^{M I}\right)}{\partial \alpha_{m, n}^{M I}}-2 \cdot \frac{\partial D_{1212}^{H}\left(\mathbf{u}_{m}^{M I}, \Phi_{m}^{M I}\right)}{\partial \alpha_{m, n}^{M I}}\right)
\end{aligned}
$$

where the macroscale displacement fields $\mathbf{u}_{m}^{M A}$ are solved by the FE analysis. The FE calculation at macro scale requires the material properties of the microstructures at all layers. On the other hand, the macroscale displacement fields are required to update the topologies of the microstructures. This indicates that $m$ sub optimization problems are required to be solved concurrently.

Similarly, the sensitivity of the volume constraint with respect to the design variables can be written by:

$$
\frac{\partial G_{m}}{\partial \alpha_{m, n}^{M I}}=\int_{\Omega_{m}^{M I}} \varphi_{m, n}^{M I}(\mathbf{x}) \delta\left(\Phi_{m}^{M I}\right) d \Omega_{m}^{M I}
$$

\subsection{Topology Optimization Algorithm}


It is noted that the bounds $\tilde{\alpha}_{m, \min }^{M I} \leq \tilde{\alpha}_{m, n}^{M I} \leq \tilde{\alpha}_{m, \max }^{M I}$ in $\left(17\right.$ ) refer to the regularized design variables $\tilde{\alpha}_{m, n}^{M I}$ rather than the actual design variables $\alpha_{m, n}^{M I}$. The actual design variables are the expansion coefficients in the CSRBE interpolation but it is not easy to specify their fixed upper and lower bounds during the optimization. Hence to facilitate the numerical implementation. the well-established OC method [33.37] is first used to undate the regularized design variables $\tilde{\alpha}_{m, n}^{M I}$ with the fixed upper and lower bounds. Then the actual design variables $\alpha_{m, n}^{M I}$ are renewed by using the updated $\tilde{\alpha}_{m, n}^{M I}$ The maior steps during the optimization can be concisely summarized, as follows:

Step 1 With the Lagrange multipliers $\Lambda_{m} \lambda_{m}^{1}$ and $\lambda_{m}^{2}$ the Lagrangian function for the optimization formulation (17) can be written as

$$
L_{m}=J_{m}+\Lambda_{m} G_{m}+\lambda_{m}^{1}\left(\tilde{\alpha}_{m, \min }^{M I}-\tilde{\alpha}_{m, n}^{M I}\right)+\lambda_{m}^{2}\left(\tilde{\alpha}_{m, n}^{M I}-\tilde{\alpha}_{m, \max }^{M I}\right)
$$

Step 2 Calculating the regularized design variables:

$$
\tilde{\alpha}_{m, n}^{M I I)}=\frac{\alpha_{m, n}^{M I I(\gamma)}-\alpha_{m, \min }^{M I(\gamma)}}{\alpha_{m,(\gamma)}^{M I(\gamma)}-\alpha_{m, \min }^{M I}}
$$

where $\gamma$ denotes the iteration number starting from 1 . $\alpha_{m, \text { min }}^{M I(\gamma)}$ and $\alpha_{m, \text { max }}^{M I(\gamma)}$ are defined by:

$$
\alpha_{m, \min }^{M I(\gamma)}=2 \times \min \left(\alpha_{m, n}^{M I(\gamma)}\right) ; \alpha_{m, \max }^{M I(\gamma)}=2 \times \max \left(\alpha_{m, n}^{M I(\gamma)}\right)
$$

Step 3 Based on the Kuhn-Tucker conditions $\left[33\right.$ 37d the regularized design variables, $\tilde{\alpha}_{m, n}^{M I}$ can be iteratively updated by using a heuristic scheme, which is given by

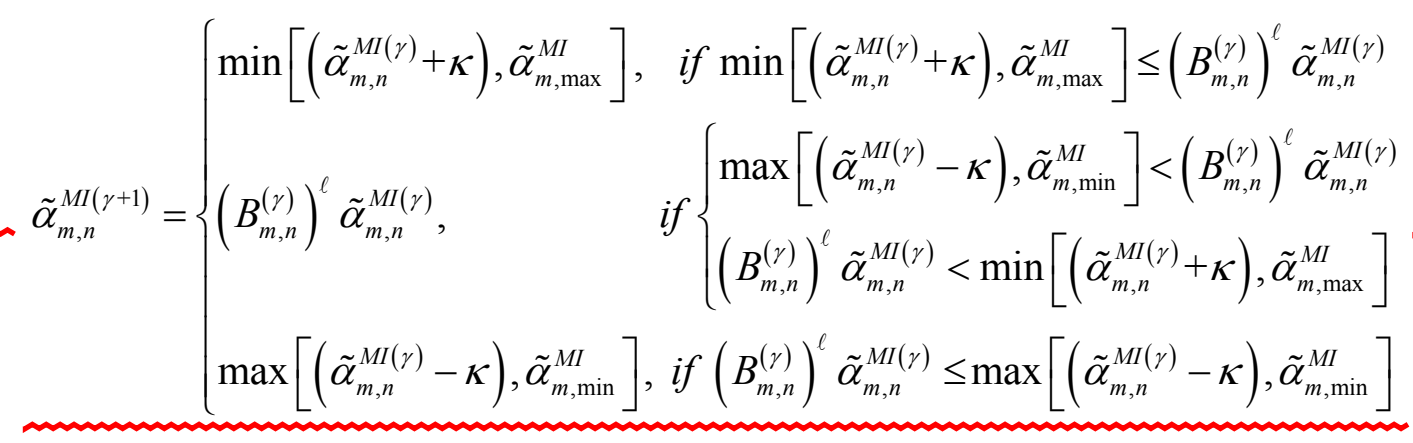

where the moxing limit $\kappa(0<\kappa<1)$ and the damping exponent $\ell(0<\ell<1)$ are the parameters [33 37 , 61l to stabilize the iteration. $\tilde{\alpha}_{m, \min }^{M I}=0.001$ and $\tilde{\alpha}_{m, \max }^{M I}=1$ are the fixed bounds on $\tilde{\alpha}_{m, n}^{M I}$ Considering $\tilde{\alpha}_{m, n}^{M I}$ is obtained by using a linear function (52) associated with $\alpha_{m, n \lambda}^{M I} B_{m, n}^{(\gamma)}$ can be defined by:

$$
B_{m, n}^{(\gamma)}=-\frac{\partial J_{m}}{\partial \alpha_{m, n}^{M I(\gamma)}} / \max \left(\mu, \Lambda_{m}^{(\gamma)} \frac{\partial G_{m}}{\partial \alpha_{m, n}^{M I(\gamma)}}\right)
$$


where $\mu$ is a small positive constant used to avoid the zero terms, and the Lagrange multipliers $\Lambda_{m}^{(\gamma)}$ can be updated by a bi-sectioning algorithm [33].

Step 4 Computing the actual design yariables:

$$
\alpha_{m, n}^{M I(\gamma+1)}=\tilde{\alpha}_{m, n}^{M I(\gamma+1)} \times\left(\alpha_{m, \text { max }}^{M I(\gamma)}-\alpha_{m, \min }^{M I(\gamma)}\right)+\alpha_{m, \min }^{M I(\gamma)}
$$

Step 5 Repeating Step 2 to Step 4 until the convergent criterion is satisfied.

\section{Numerical implementation}

In the numerical implementation, one of the issues is the representation of discontinuities when the elements are cut by a moving boundary. In this study, the strains of those elements are approximated by using the "ersatz material" model $[42,61]$ without remeshing. The "ersatz material" model uses a weak material to fill the void of the design domain, and thus the density, strain and stiffness of an element are assumed to be proportional to its area fraction. For more details, the readers may refer to [42, 61].

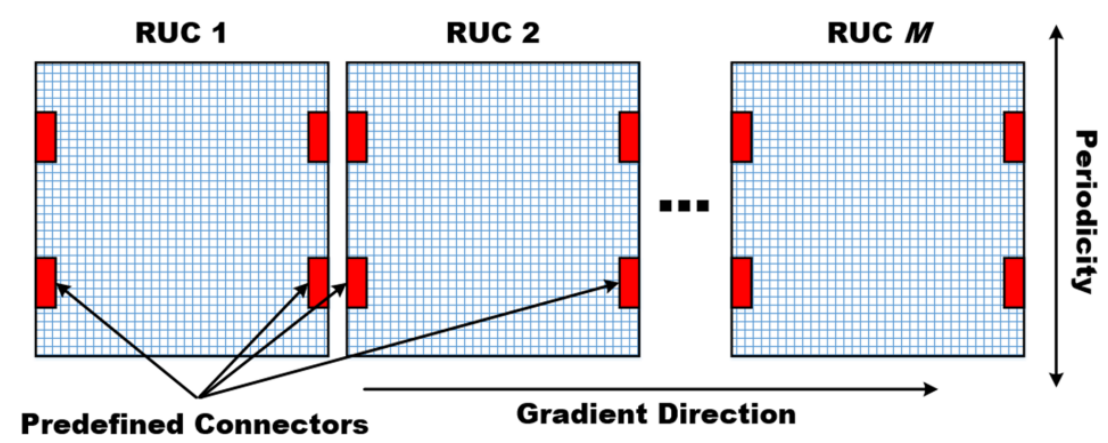

Figure 3. Kinematical connectivity between different RUCs (representative unit cells)

In conventional homogeneous material design, the periodic boundary conditions are applied to microstructures, and there is no connectivity issue between adjacent microstructures. However, in FGM designs, the periodicity for those microstructures along the functionally graded direction will be violated in order to allow for the gradient in the property of the bulk material. In this case, the adjacent microstructures between different layers may not be connected properly. Thus, the connectivity of the adjacent microstructures in the FGMs is essential for generating a feasible FGM design. In this paper, a kinematical connectivity method as shown in Fig. 3 is adopted to tackle this issue. Readers can refer to [25] for more about the kinematical connectivity method. This method introduces several prescribed connectors in the 
adjacent unit cells along the property gradient direction, and these connectors are kept as identical as possible to maintain numerical accuracy, as well as periodicity [25]. In the design, these pre-defined connectors can be fulfilled by setting the non-design regions in the design domain.

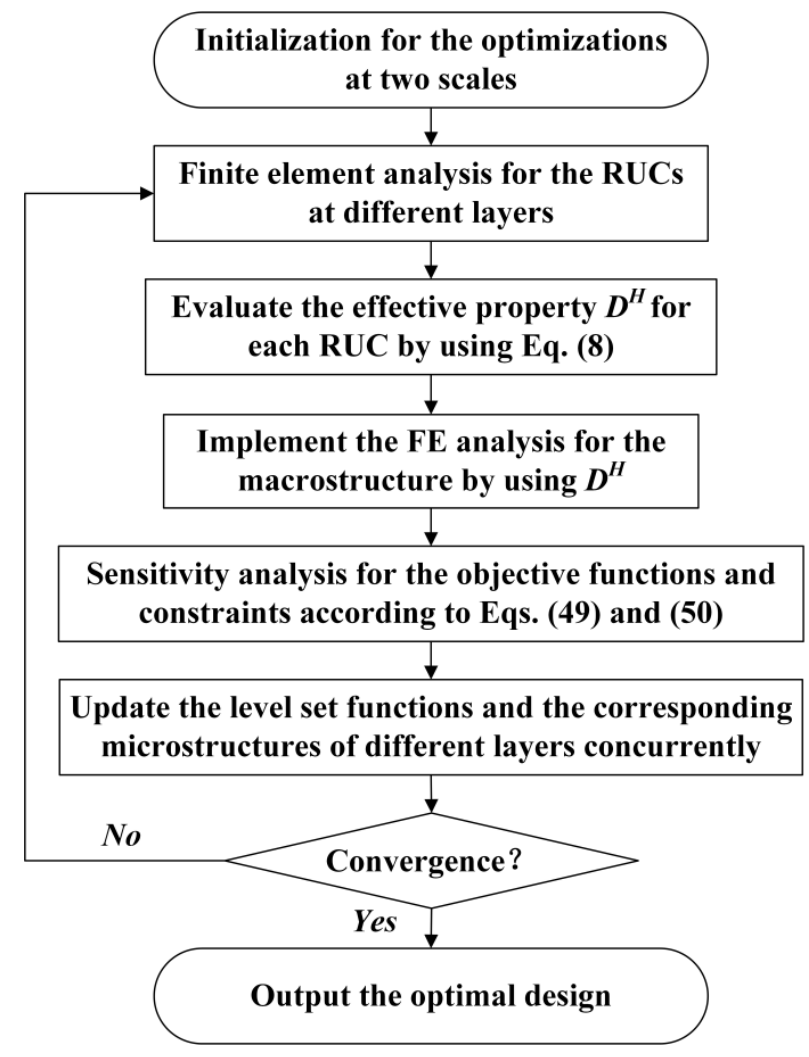

Figure 4. Flowchart of the numerical implementation for the proposed method

The flowchart of the proposed method is shown in Fig. 4. The micro FE analysis is first implemented at each layer, and then the effective material properties of microstructures are calculated by using the numerical homogenization method, given in Eq. (8). Then, another FE analysis at the macroscale, based on the effective properties obtained from the micro FE analysis at different layers, is used to calculate the displacement field and the mean compliance of the macrostructure. For the sub-optimization problem defined at each layer, the derivatives of the objective function and the volume constraint are obtained via Eq. (49) and Eq. (50). With the sensitivity information, the OC-based algorithm is employed to update the design variables and the corresponding level set functions over each layer, so as to implicitly optimize the microstructures. It is noticed that all the microstructural optimization problems at different layers are solved concurrently, because all the microstructures serve as a whole to increase the overall stiffness of the macrostructure. The optimization process repeats until the optimized designs are achieved. 


\section{Numerical Examples}

In this paper, several numerical examples are used to demonstrate the proposed method for devising the FGM composites with auxetic metamaterials. Both the isotropic and orthotropic material microstructures are considered by applying the geometrical symmetry to the RUCs. In the numerical process, the isotropy is achieved as follows: the square and diagonal geometrical symmetries are applied to the 2D microstructure (a total of 4 symmetries) [50]. For the orthotropy of 2D cases, we apply two symmetries with respect to $x$ and $y$ directions to the microstructure.

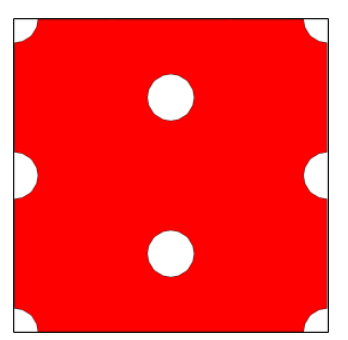

(a) Orthotropy

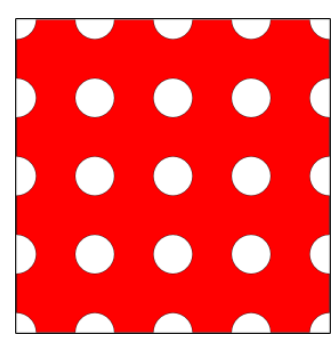

(b) Isotropy

Figure 5. Initial designs for the orthotropic and isotropic material microstructures

In all examples, the Young's modulus of the solid material is 180, and the Poisson's ratio is 0.3 . The materials are subject to plane stress conditions. The finite element method is applied to both the macro and micro scales to discretize the design domains. For simplicity, the size of a RUC (or microstructure) is assumed to be the same as that of a macro finite element. All the numerical cases for the representative microstructures at all layers are optimized by the PLSM, and no further upwind schemes, re-initializations and velocity extensions are required like most conventional LSM methods [41-43]. In each microstructure, we assume that the level set grid is the same as the FE mesh for simplicity. It is noted that the numerical homogenization method is used to evaluate the effective property of the material microstructure, and the full periodicity assumptions are enforced on all the microstructures.

In Fig. 5, two different initial designs are provided in order to achieve different microstructural designs with the orthotropic and isotropic material properties, respectively. In the inverse design of material microstructures, it is noted that the same elastic property to be achieved may correspond to a number of different microstructures. Hence, the different initial designs of the microstructures may only affect the 
final topology of the design rather than its effective material property $[44,50]$. For simplicity, we use the same weight parameters $w_{m, a}$ and $w_{m, b}$ for each sub-optimization problem. The optimization terminates when the relative difference of the summation of all the $M$ objective function values between two successive iterations is less than 0.0002 , or the maximum 300 steps are reached.

\subsection{Structure with a concentrated force}

Fig. 6 shows a structure with length $\mathrm{L}=30 \mathrm{~cm}$ and height $\mathrm{H}=10 \mathrm{~cm}$ subject to a concentrated force $\mathrm{F}=50 \mathrm{~N}$ at the center point of the lower edge, and the upper edge is fixed. The macrostructure is divided into 10 layers, and 10 sub-optimization problems are to be solved. The gradient direction is assumed to be along the $y$ axis, with an increase of the volume fractions from the top layer to the bottom layer.

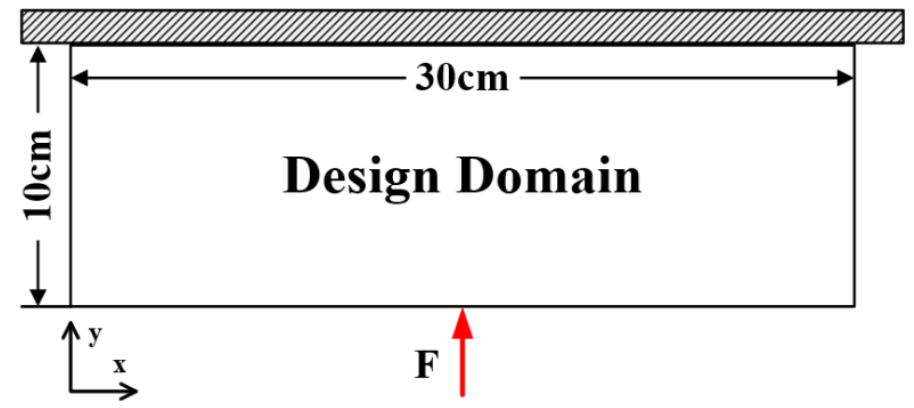

Figure 6. Design domain with a single load

\subsubsection{Numerical comparison}

In this example, two typical numerical cases are used to exhibit the features of the topologically designed FGM using the proposed method. Here, orthotropic material microstructures are considered. The macro structure is discretized by $30 \times 10=300$ four-node quadrilateral (Q4) elements, and microstructures at different layers are all discretized by $40 \times 40=1600$ Q 4 elements. To enable the FGM property, the volume fractions for different layers are restrained to change nonlinearly from 0.25 to 0.55 along the gradient direction (increased from top to bottom). If $\xi=2$ used in Eq. (22), the volume constraints of the 10 layers are $0.25,0.2537,0.2648,0.2833,0.3039,0.3406,0.3833,0.4315,0.4870$ and 0.55 , respectively. The total volume fraction of the macro composite can be averaged as 0.3554 .

For comparison, the macrostructure is also assumed to be composed of uniform microstructures (without gradient properties). For the design uniformly configured with an array of identical microstructures, the total volume fraction constraint is also 0.3554 which is equal to the volume of the FGM-based composite. 
To facilitate the comparison, two optimization problems with single objective functions are implemented to optimize either the structural stiffness or the negative Poisson' ratio.

The FGM-based designs are given in Fig. 7, and the designs with the uniform microstructures are shown in Fig. 8. It can be seen that all the optimized designs are characterized with smooth structural boundaries and distinct material interfaces due to the use of the level set-based method. Furthermore, the final topologies of the microstructures in Fig. 7(a) and Fig. 8(a) are similar to the widely accepted re-entrant type auxetic metamaterials in this field $[5,6]$.

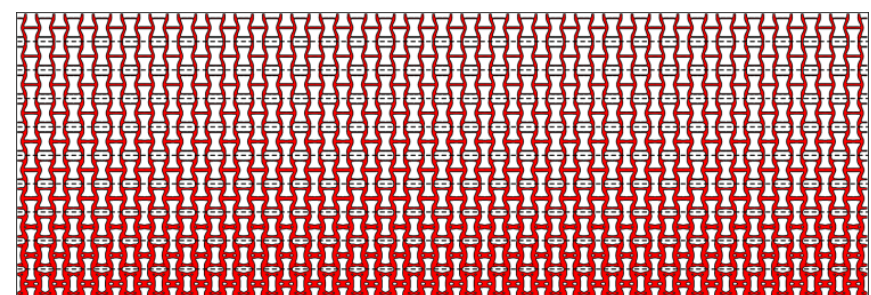

(a)

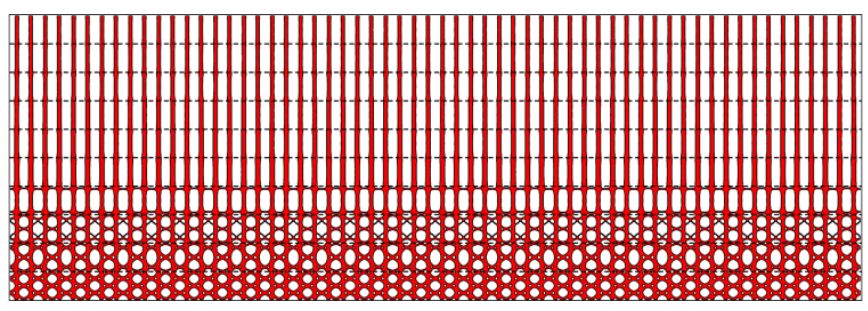

(b)

Figure 7. FGM-based composites: (a) complete NPR design, where averaged $v_{12}$ is -1.1725 , averaged $v_{21}$ is -0.2896 and mean compliance is 182.2 ; (b) complete mean compliance optimization design, where averaged $v_{12}$ is 0.2826 , averaged $v_{21}$ is 0.0691 and mean compliance is 73 .

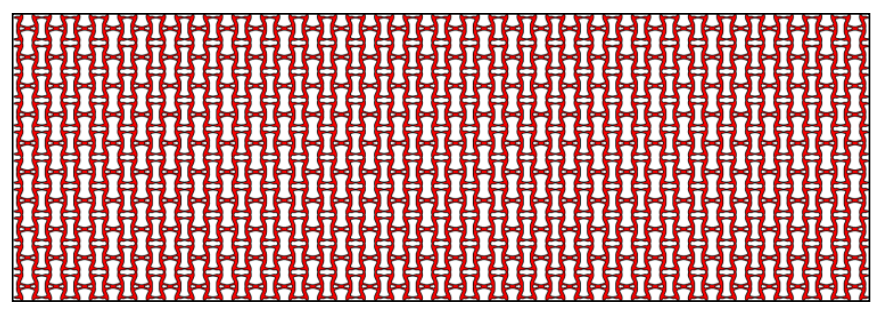

(a)

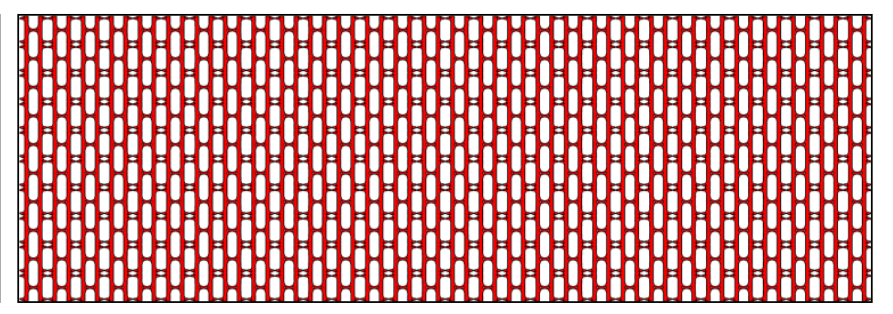

(b)

Figure 8. Optimal designs with uniform microstructures: (a) complete NPR optimization design, where $v_{12}$ is $-1.128, v_{21}$ is -0.2973 and mean compliance is 303.5 ; (b) complete mean compliance optimization design, where $v_{12}$ is $0.6798, v_{21}$ is 0.0815 and mean compliance is 135.7 .

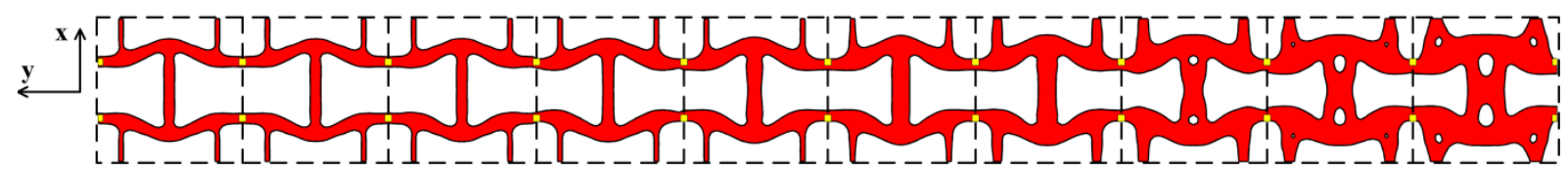

Figure 9. Non-designable connectors for the orthotropic material microstructures

It is noted that the Poisson's ratios $v_{12}$ and $v_{21}$ for orthotropic material may be different. $v_{12}$ and $v_{21}$ of each composite layer with orthotropic material microstructures can be approximated by the numerical 
homogenization method. However, for a FGM-based macrostructure that contains a number of different microstructures, it is difficult to find an analytical method to quantify the overall Poisson's ratios $v_{12}$ and $v_{21}$ of the macrostructure. In this paper, we average all the effective Poisson's ratios $v_{12}$ of microstructures at different layers within a macrostructure to approximate the overall Poisson's ratio $v_{12}$ of the macrostructure. Similarly, the macrostructural Poisson's ratio $v_{21}$ can be approximated.

We further compare the results in Figs. 7 and 8. On one hand, in the complete stiffness design, the FGMbased composite is stiffer than the design only with the uniform microstructures. On the other hand, for the pure NPR design, the FGM composite shows a better averaged $v_{12}$ and a similar averaged $v_{21}$ when comparing with the design with uniform microstructures. Furthermore, if the same loading and boundary conditions are applied, the FGM-based composite can have a better stiffness due to the graded material distribution. These show that the FGM-based composites have better performance than the traditional designs with an array of identical microstructures.

From Fig. 9, it can be found that the microstructural connectivity of the FGM-based composite is well preserved due to the introduction of the non-designable connectors (the yellow square regions) between the adjacent microstructures. It is also noted that the Poisson's ratio for 2D orthotropic materials along a graded direction theoretically can be lower than -1 that is also the lower bound for $2 \mathrm{D}$ isotropic microstructure [14]. This indicates that the orthotropic material microstructure can explore a better NPR property along a specified direction.

\subsubsection{Effect of weighting coefficients}

Here, for the multi-objective formulation defined in Eq. (17), several weight coefficients have been implemented to show the different design requirements for structural stiffness (mean compliance) and auxetic behavior of the microstructure. In these cases, the FE models and the volume gradient are exactly the same as in the examples given in Fig. 7. The optimal results with different weight coefficients are given in Fig. 10. Correspondingly, Fig. 11 shows the variation of material properties over different layers, including the Poisson's ratios $v_{12}, v_{21}$ and volume fraction of the microstructure at each layer, as well as the mean compliance of each layer. The iterations of the four cases for convergence are 161, 145, 109 and 100. 
In the four cases, the non-designable connectors between the adjacent microstructures are also defined as shown in Fig. 9, and thus the connectivity of each FGM-based composites is well preserved.

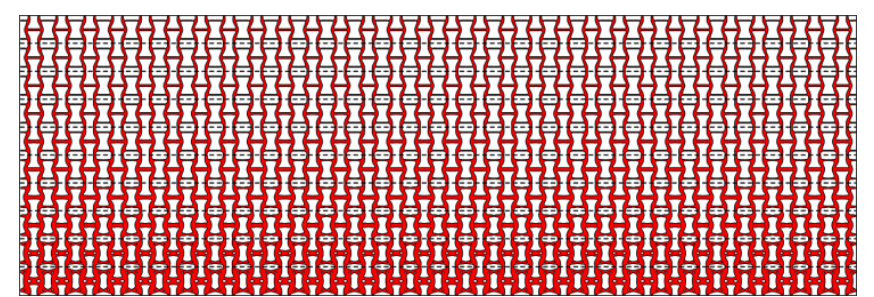

(a) $w_{m, a}=0.2, w_{m, b}=0.8: f^{M A}=176.5$

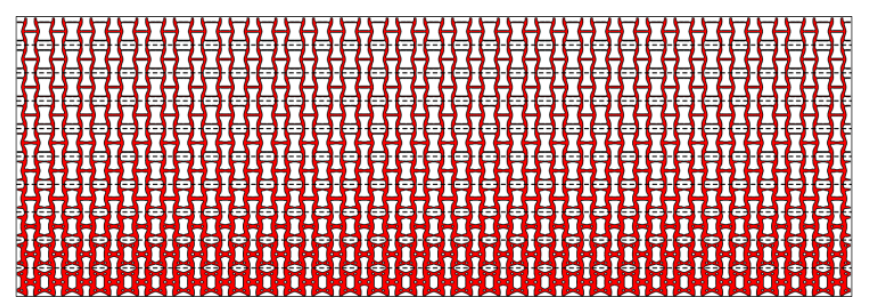

(c) $w_{m, a}=0.6, w_{m, b}=0.4: f^{M A}=134.3$

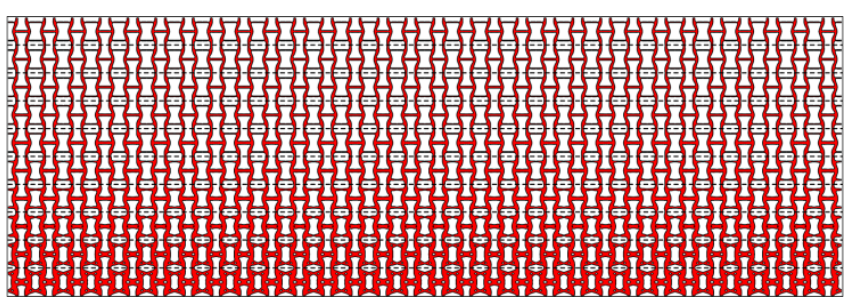

(b) $w_{m, a}=0.4, w_{m, b}=0.6: f^{M A}=157.9$

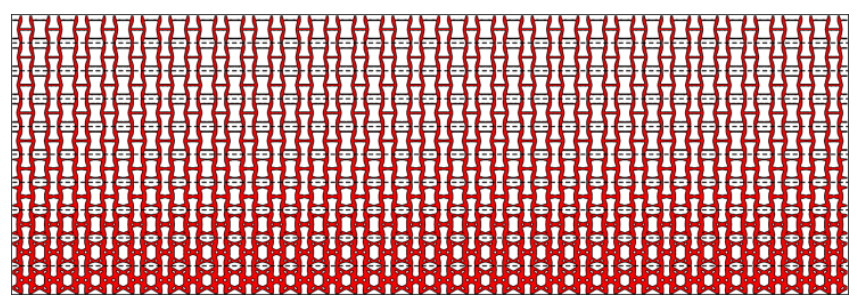

(d) $w_{m, a}=0.8, w_{m, b}=0.2: f^{M A}=109.4$

Figure 10. Optimal composites with different weight coefficients

To facilitate the discussion, the averaged $v_{12}$ and $v_{21}$ of all layers are also presented to approximate the NPR property of the entire composite. For the four cases in Fig. 11, the averaged $v_{12}$ are $-1.147,-1.1186,-1.0694$ and -0.9556 , respectively. While the averaged $v_{21}$ are $-0.2879,-0.2608,-0.2161$ and -0.1486 , respectively. Obviously, when the weight $w_{m, a}$ increases, a larger weight is given to the macro objective function $f^{M A}$, and the overall structural stiffness improves gradually. On the contrary, the averaged $v_{12}$ and $v_{21}$ of the composites increase (NPR properties decrease). This implies that the multi-objective formulation indeed makes a tradeoff between structural stiffness (or mean compliance) and NPR. Actually, a larger weight on the mean compliance is not preferable, as it may limit the NPR properties for all the microstructures inside the design domain. As seen in Figs. 10(d) and 11(d), one layer contains microstructures that fail to exhibit mechanism-type topologies [14], and does not show auxetic behavior.

Furthermore, Fig. 11 shows that all the volume constraints are satisfied, and there is a nonlinear increase of the volume fractions from layer 1 to 10 . In engineering, the layers that are closer to the external load require more materials and denser microstructures to resist the deformation. On the other hand, as shown in Figs. 11(a) - (c), microstructures with lower densities exhibit better auxetic behavior. To satisfy the 
multifunctional requirements, the volume gradient constraints for the FGMs are increased from layer 1 to layer 10, where the higher-density material microstructures are mainly used to enhance the stiffness while the lower-density material microstructures are devised to guarantee the NPR property.

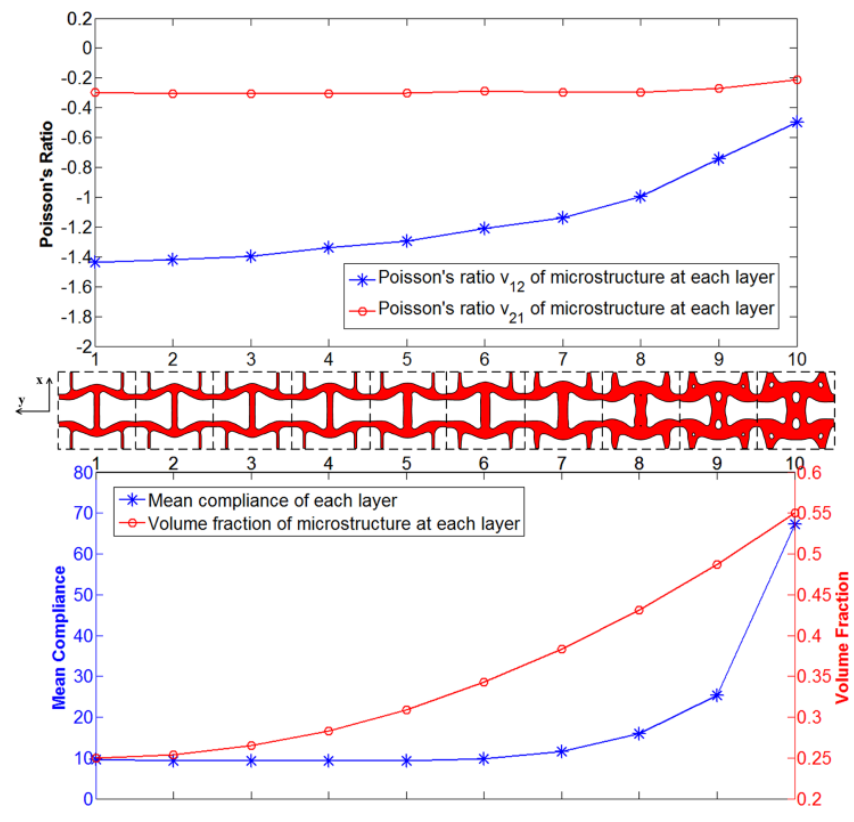

(a) $w_{m, a}=0.2$ and $w_{m, b}=0.8$

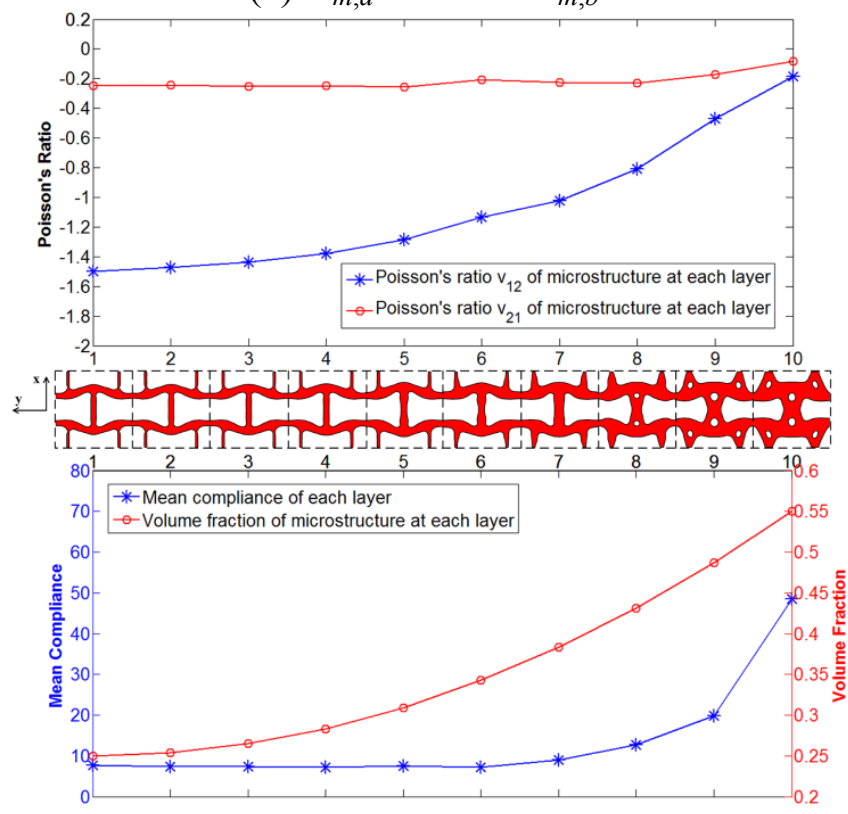

(c) $w_{m, a}=0.6$ and $w_{m, b}=0.4$

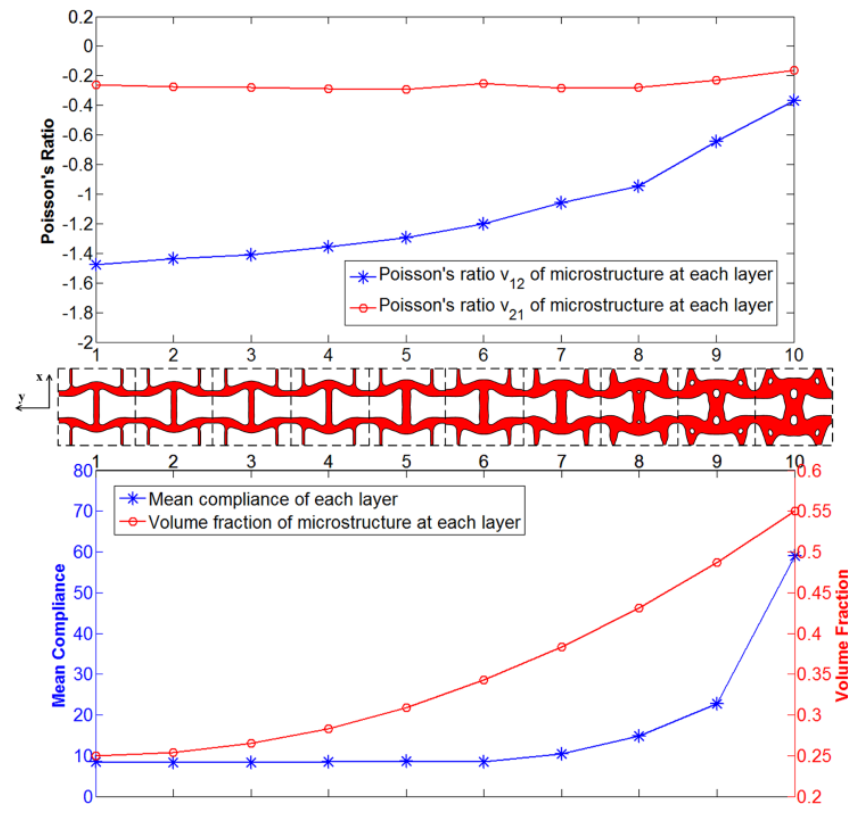

(b) $w_{m, a}=0.4$ and $w_{m, b}=0.6$

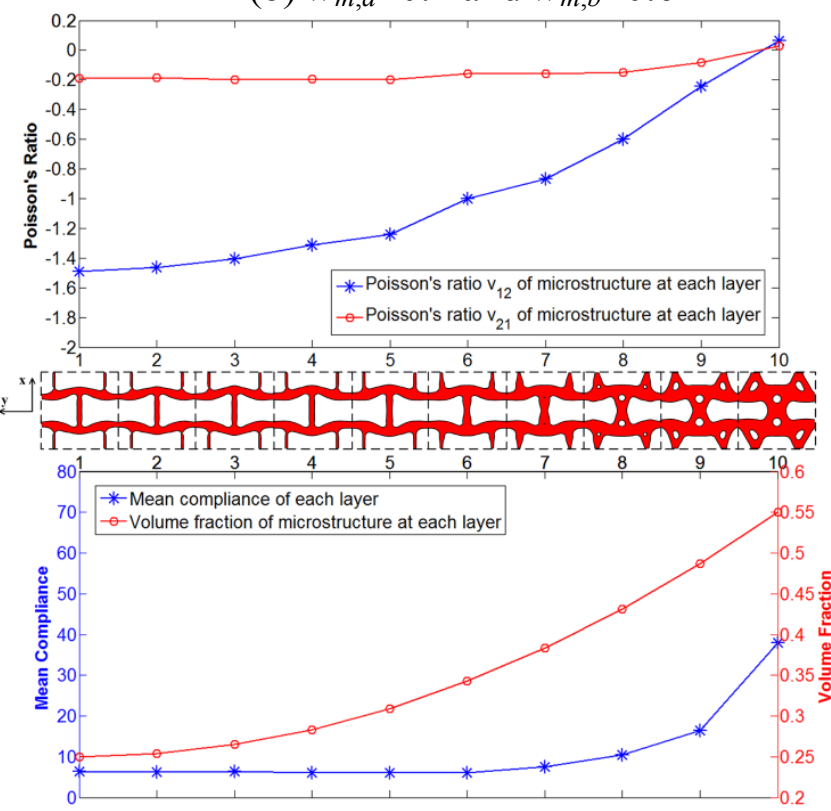

(d) $w_{m, a}=0.8$ and $w_{m, b}=0.2$

Figure 11. Optimal results with the different weight coefficients

\subsubsection{Investigation of the isotropic materials}

Here, the isotropic material microstructures are considered. The design domain is shown in Fig. 6, and the macrostructure is discretized by $30 \times 10=300$ Q 4 elements, while the microstructures are discretized by 
$80 \times 80=6400 \mathrm{Q} 4$ elements. The weights for the objectives are $w_{m, a}=0.2$ and $w_{m, b}=0.8$, respectively. The volume gradient constraint nonlinearly changes from 0.35 to 0.65 , and the coefficient $\xi=2$.

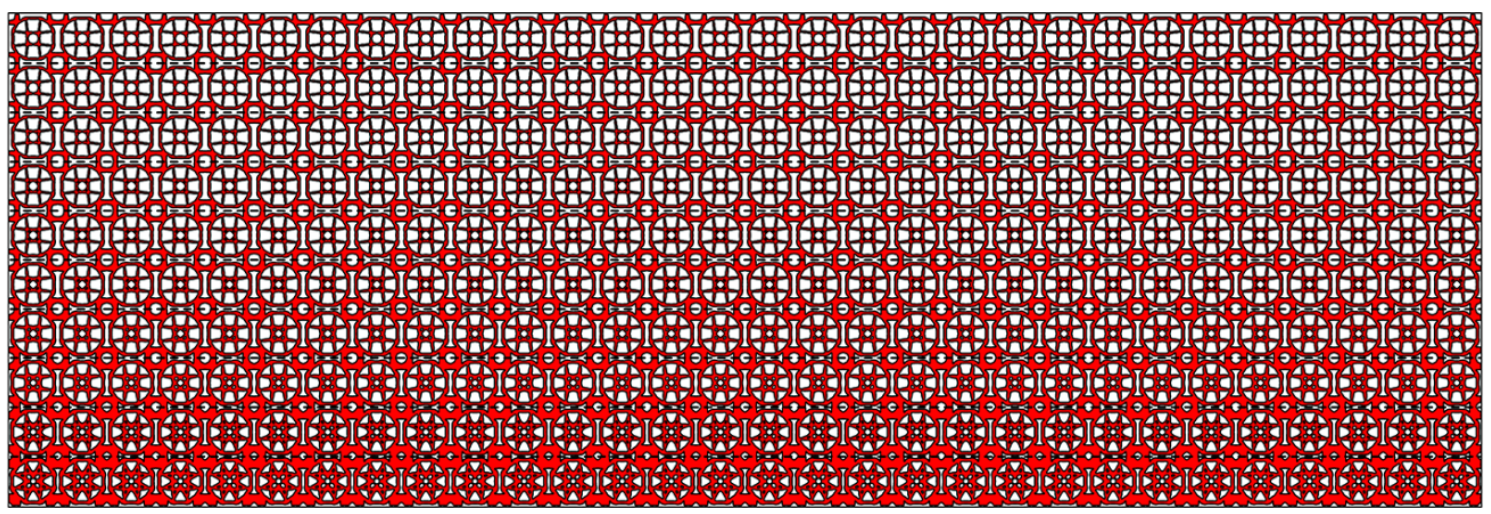

Figure 12. Optimal composite structure with isotropic FGMs

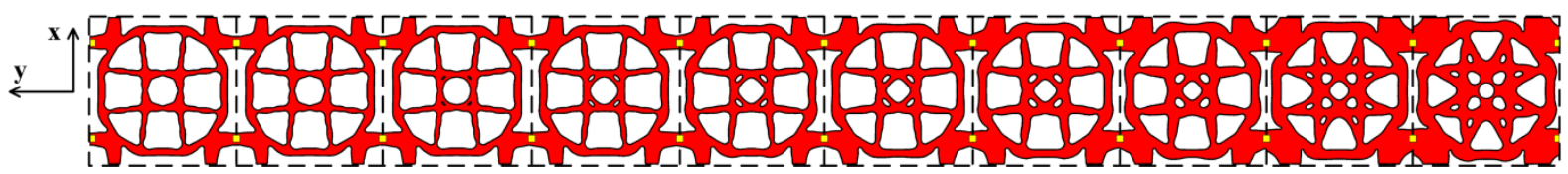

Figure 13. Non-designable connectors for the isotropic materials

The initial design of the microstructure with square and diagonal symmetries is given in Fig. 5(b). The optimized composite structure is given in Fig. 12, with the mean compliance equal to 121.2. The topological differences and the material properties over layers are shown in Table 1 . We can find that the connectivity between different layers is well preserved, due to the introduction of the non-designable connectors between different layers (see the yellow square regions in Fig. 13). Along the gradient direction (from top to bottom), the volume gradient is satisfied, and the material properties as well as the mean compliances over different layers change gradually. It can be found that the proposed method can design FGMs with NPRs not only for orthotropic materials but also for isotropic materials.

The convergent histories are plotted in Figs. 14(a) and (b). It takes 152 iterations to achieve the optimized design. It can be seen that during the first few steps (about 10 iterations), the objective function increases because the volume constraints are not fully satisfied at this initial stage of optimization. The objective functions of the sub optimization problems increase rather than reduce, and materials are being removed from the microstructures until the constraints are satisfied. Once the volume constraints are satisfied and 
become active, the objective functions start going down steadily and the optimization is being iteratively processed until the convergence criterion is met.

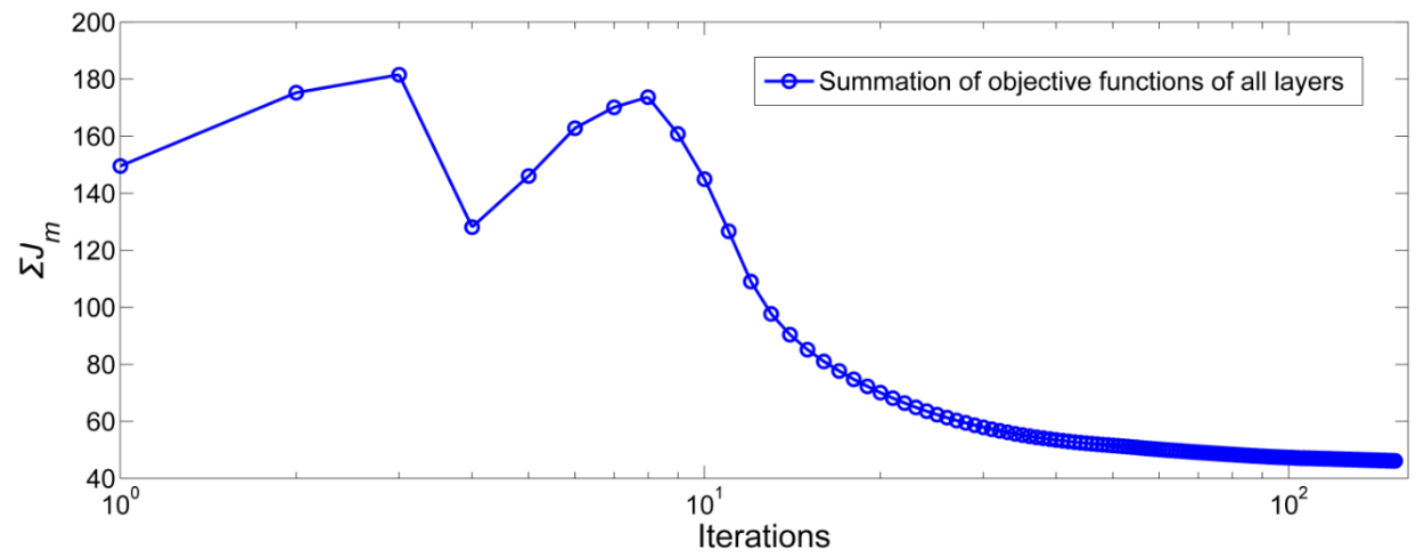

Figure 14(a). Iterative histories: summation of objective functions of all layers

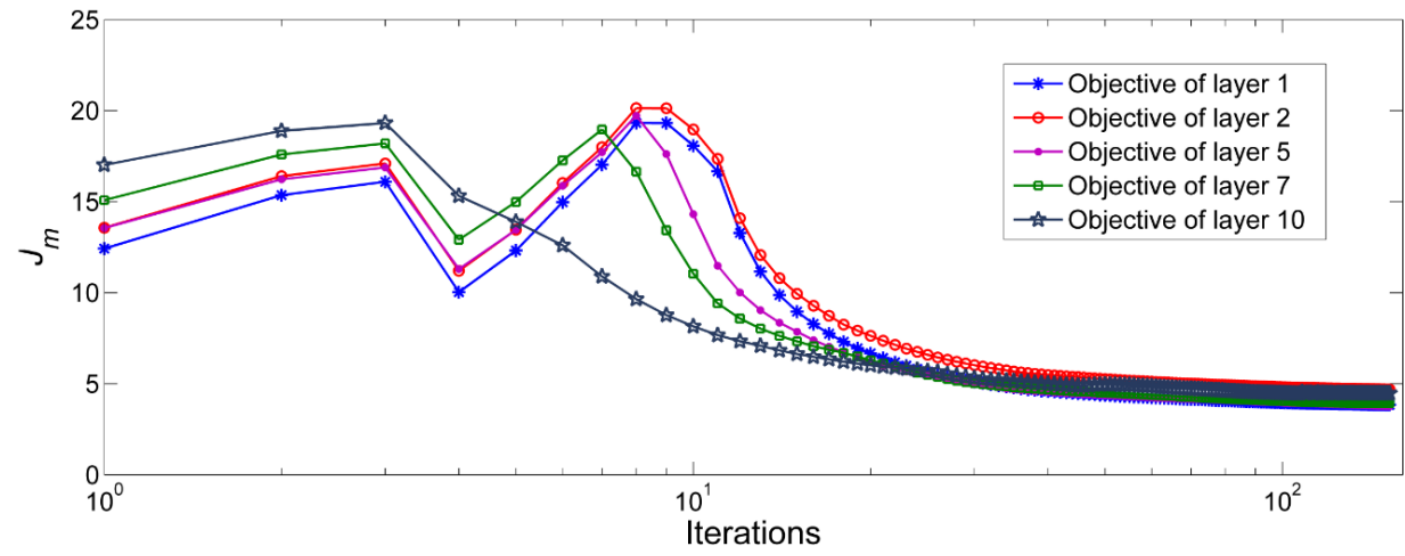

Figure 14(b). Iterative histories: objective functions of some layers

Table 1. Optimized properties of the isotropic FGMs

\begin{tabular}{|c|c|c|c|c|c|c|c|c|}
\hline \multirow{3}{*}{$\begin{array}{c}\text { Layer } \\
1\end{array}$} & \multicolumn{8}{|c|}{ Isotropic FGMs } \\
\hline & \multirow{2}{*}{$\begin{array}{c}\text { Volume } \\
0.3500\end{array}$} & \multirow{2}{*}{$\begin{array}{c}\text { Poisson's ratio } \\
-0.3999\end{array}$} & \multirow{2}{*}{$\begin{array}{c}\text { Mean compliance } \\
5.8336\end{array}$} & \multirow{2}{*}{$\frac{\text { Microstructure }}{100}$} & \multirow{2}{*}{ Level set surface } & \multicolumn{3}{|c|}{ Elasticity tensor } \\
\hline & & & & & & {$\left[\begin{array}{c}18.83 \\
-7.53 \\
0\end{array}\right.$} & $\begin{array}{c}-7.53 \\
18.83 \\
0\end{array}$ & $\begin{array}{c}0 \\
0 \\
1.32\end{array}$ \\
\hline 2 & 0.3537 & -0.3970 & 5.6923 & & & {$\left[\begin{array}{c}19.07 \\
-7.57 \\
0\end{array}\right.$} & $\begin{array}{c}-7.57 \\
19.07 \\
0\end{array}$ & $\left.\begin{array}{c}0 \\
0 \\
1.37\end{array}\right]$ \\
\hline 3 & 0.3648 & -0.3866 & 5.7106 & & & {$\left[\begin{array}{c}19.79 \\
-7.65 \\
0\end{array}\right.$} & $\begin{array}{c}-7.65 \\
19.79 \\
0\end{array}$ & $\begin{array}{c}0 \\
0 \\
1.61\end{array}$ \\
\hline
\end{tabular}




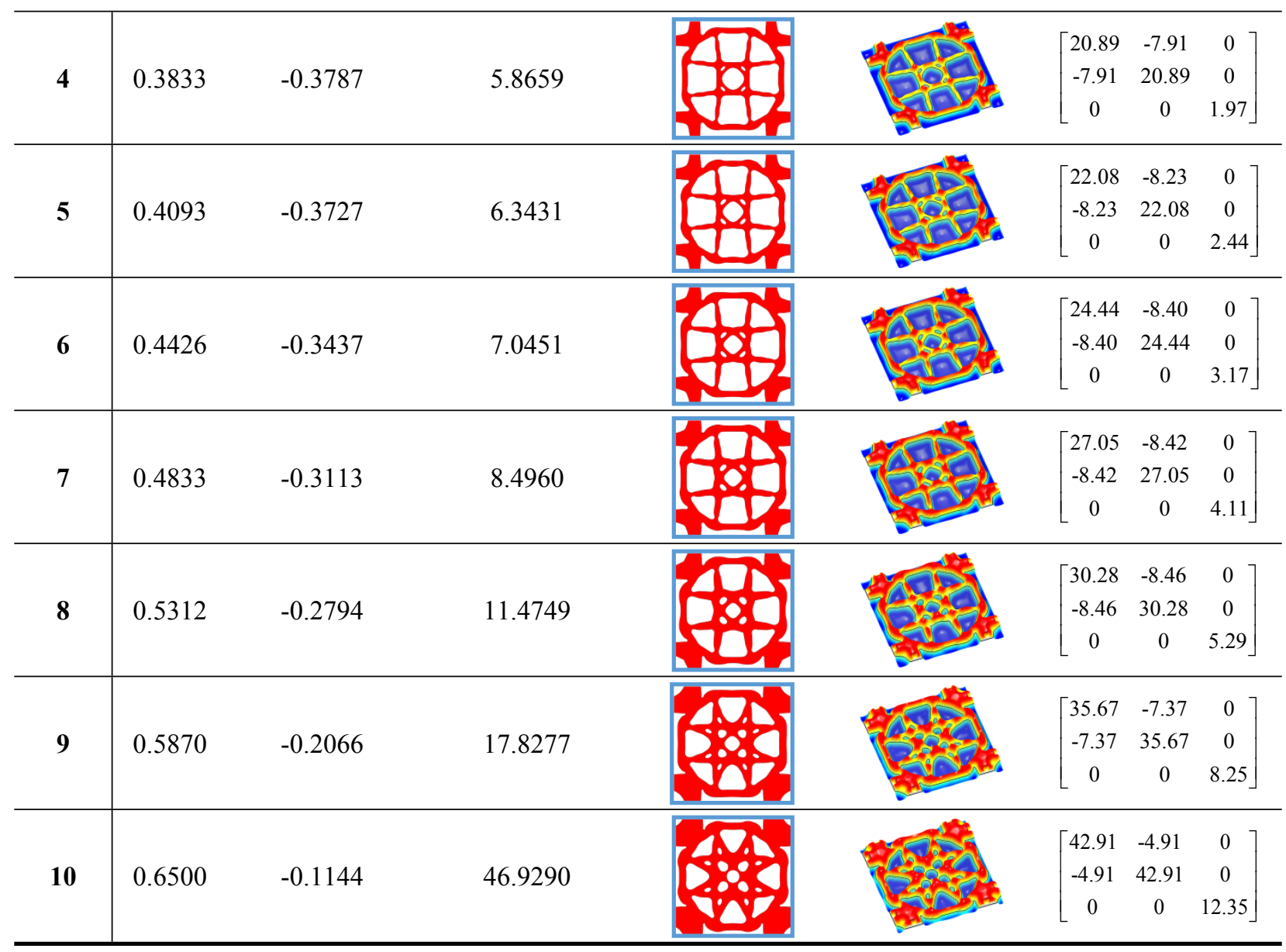

\subsection{Structure with uniformly-distributed loads}

As shown in Fig. 15 , the $10 \mathrm{~cm} \times 10 \mathrm{~cm}$ structure is fixed at the lower edge. The vertical pressure $\mathrm{F}=10 \mathrm{~N} / \mathrm{cm}$ is uniformly applied at the upper edge of the structure. In this example, the influence of different number of layers (or the dimensions of microstructures) upon the optimized design is investigated. The macro composite is divided into 10, 20 and 40 layers, respectively. In other words, the macro structure is discretized by $10 \times 10=100,20 \times 20=400$ and $40 \times 40=1600$ elements. However, the microstructures are all discretized by $40 \times 40=1600 \mathrm{Q} 4$ elements. The linear gradient volume constraints (from 0.45 to 0.25 ) are imposed to generate FGMs along the $y$ axis (from top to bottom), where $\xi=1$. Therefore, although different layers are considered, the overall volume fractions of all three cases are 0.35 . The weights of the multiobjective functions, namely the structural stiffness and NPR, are $w_{m, a}=0.2$ and $w_{m, b}=0.8$. For comparison purpose, we also design the structure with a single-objective formulation that only considers the NPR design (namely, $w_{m, a}=0$ and $w_{m, b}=1$ ). 
In these numerical cases, the initial design is still the same as in Fig. 5(a). A variety of designs have been obtained by changing the number of layers for the macro composite, as shown in Fig. 16. The corresponding microstructures and their properties over layers are shown in Fig. 17. The $v_{12}$ and $v_{21}$ for the FGM composite after averaging are given in Table 2. It is seen that the macro composite structure maintains good connectivity between different layers, due to the same non-designable connectors as in Fig. 9. It is obvious that the volume constraints are well satisfied for all different cases. Unlike the volume fractions, the mean compliances and Poisson's ratios sometimes change non-monotonously. This is because that we only impose a gradient constraint on the volumes rather than other properties.

It can be found that the FGM composites generated by the multi-objective method have better structural stiffness compared to the results obtained by the single-objective design only considering NPR. Moreover, the results denote that the FGM composites with more layers are slightly stiffer, due to the refinement of the macro FE mesh. Hence we can see that the further increase of the number of layers may have limited influence on the final design, if the size of the microstructure has already been small enough for the multiscale design. According to Eq. (22), when more layers involved in a macro structure with FGMs, smaller variation of the property gradient can be achieved. In this case, the numerical homogenization method should be more accurate in estimating the FGM properties.

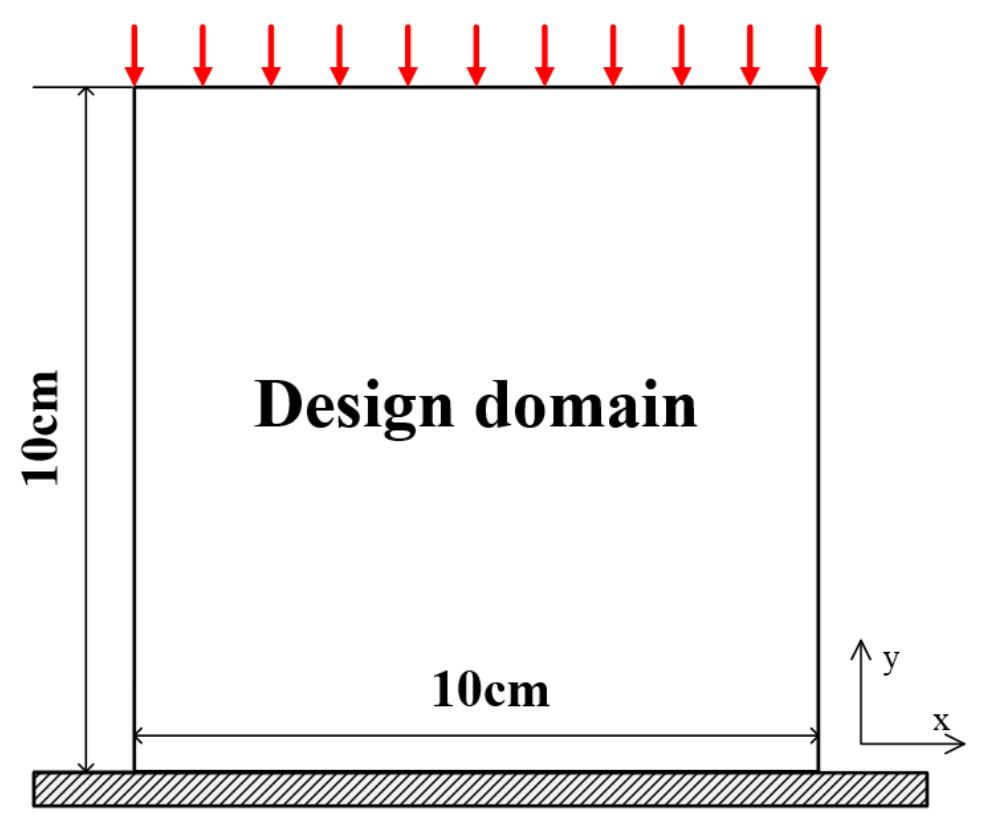

Figure 15. Design domain with uniformly-distributed loads 

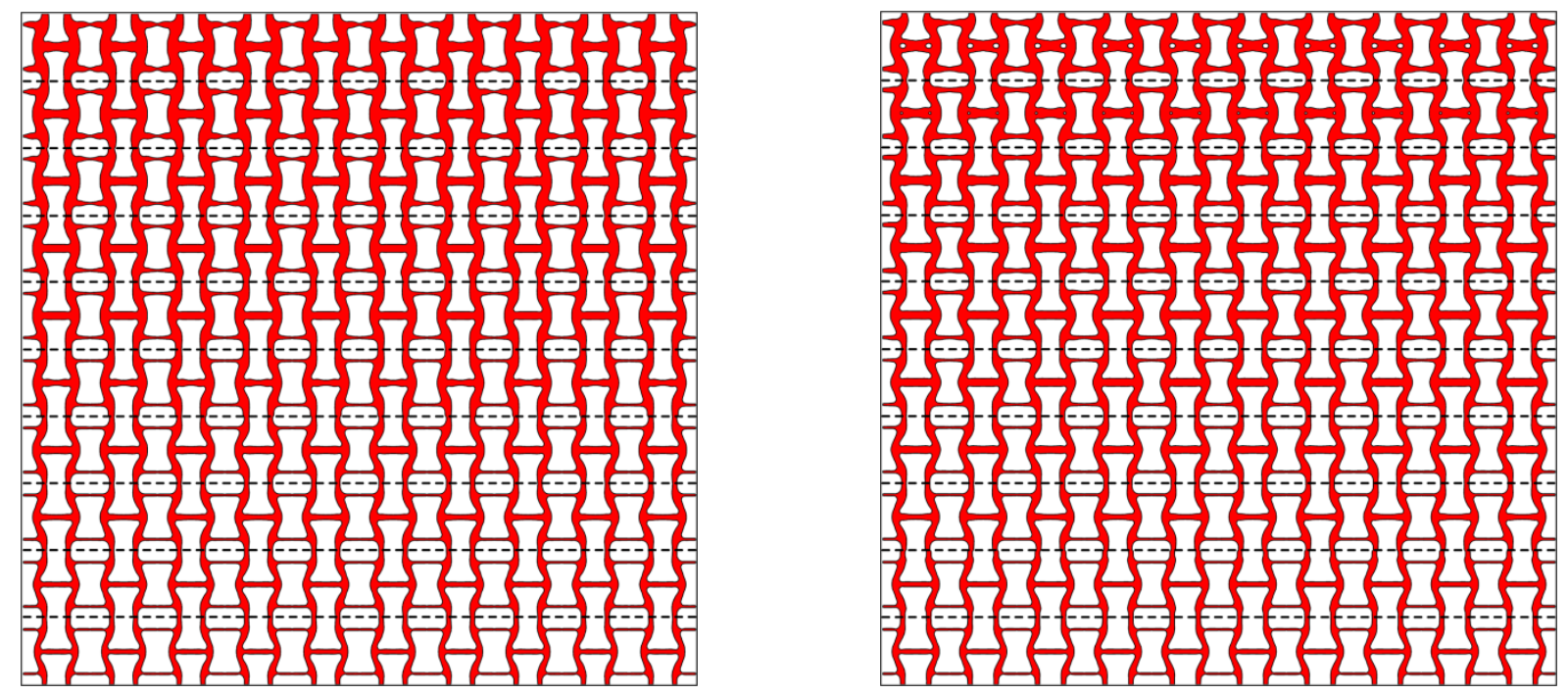

(a) 10 layers: Multi-objective design $f^{M A}=238.8$ (Left); Single-objective design $f^{M A}=302.5$ (Right)
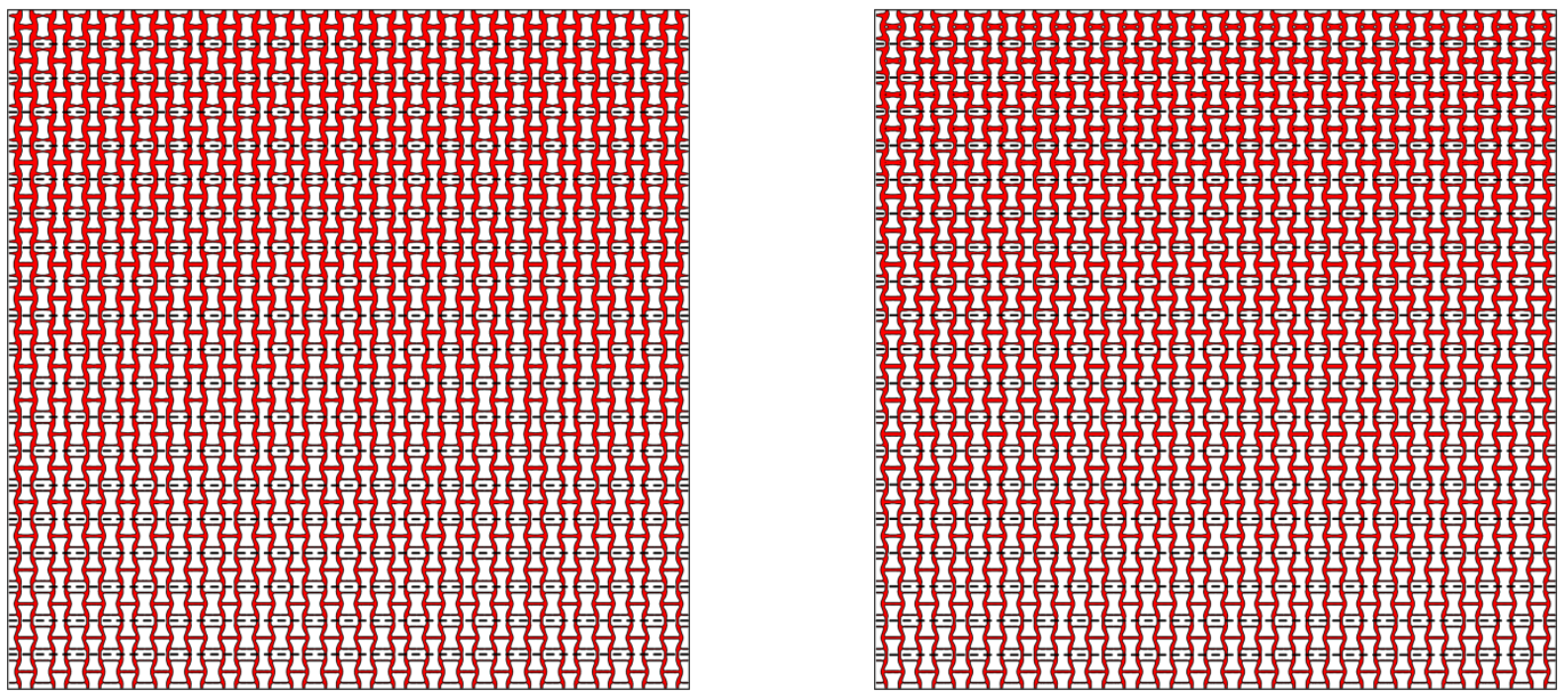

(b) 20 layers: Multi-objective design $f^{M A}=226.5$ (Left); Single-objective design $f^{M A}=294.1$ (Right)
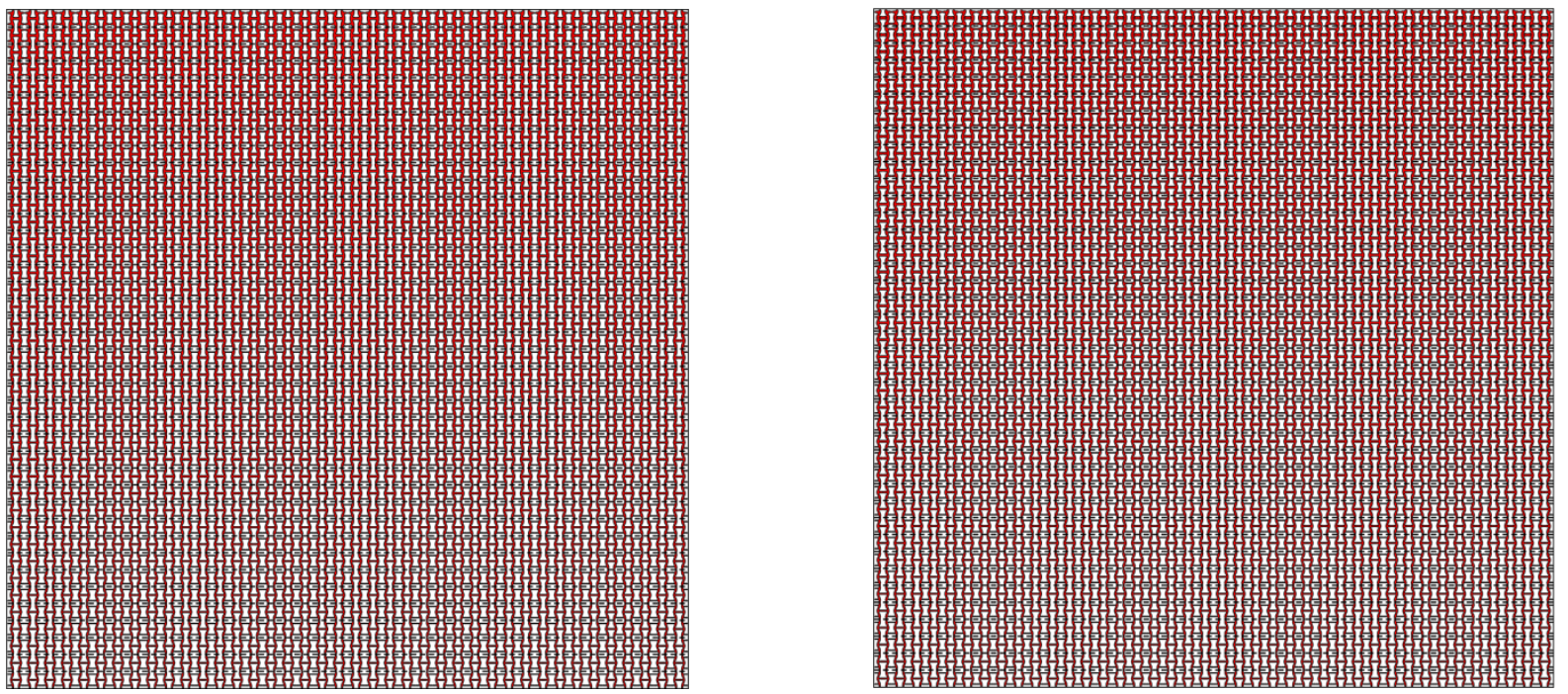

(c) 40 layers: Multi-objective design $f^{M A}=223.9$ (Left); Single-objective design $f^{M A}=291.3$ (Right) Figure 16. Designs with different layers 
Table 2. Optimized properties of the FGMs with different layers

\begin{tabular}{llll}
\hline Method & Number of Layers & Averaged $v_{12}$ & Averaged $v_{21}$ \\
\hline Multi-objective optimization & 10 & -1.1471 & -0.2402 \\
& 20 & -1.1439 & -0.2361 \\
& 40 & -1.1472 & -0.2363 \\
Single-objective optimization & 10 & -1.2047 & -0.3032 \\
& 20 & -1.2048 & -0.3034 \\
& 40 & -1.2066 & -0.3034 \\
\hline
\end{tabular}
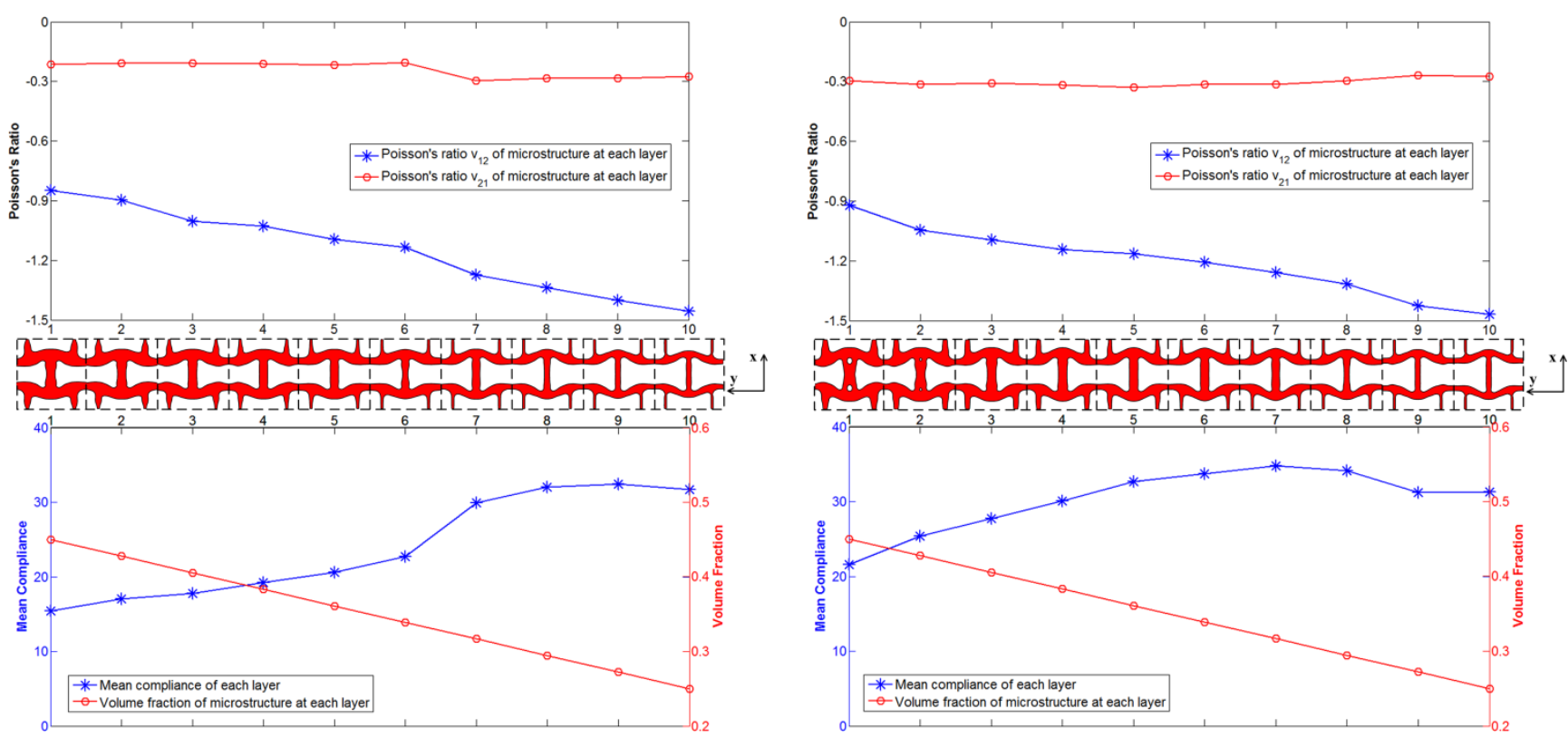

(a) Properties over 10 layers: Multi-objective design (Left); Single-objective design (Right)
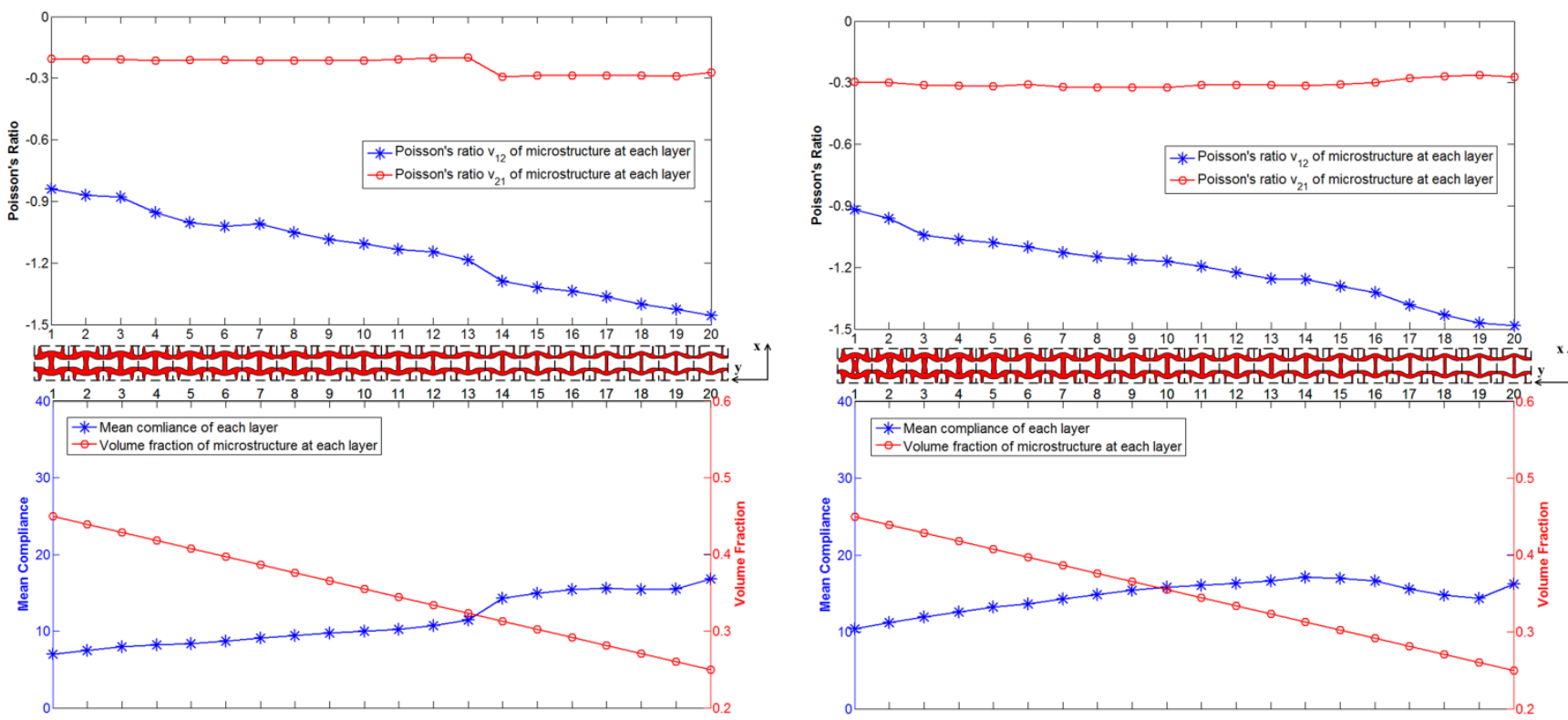

(b) Properties over 20 layers: Multi-objective design (Left); Single-objective design (Right) 

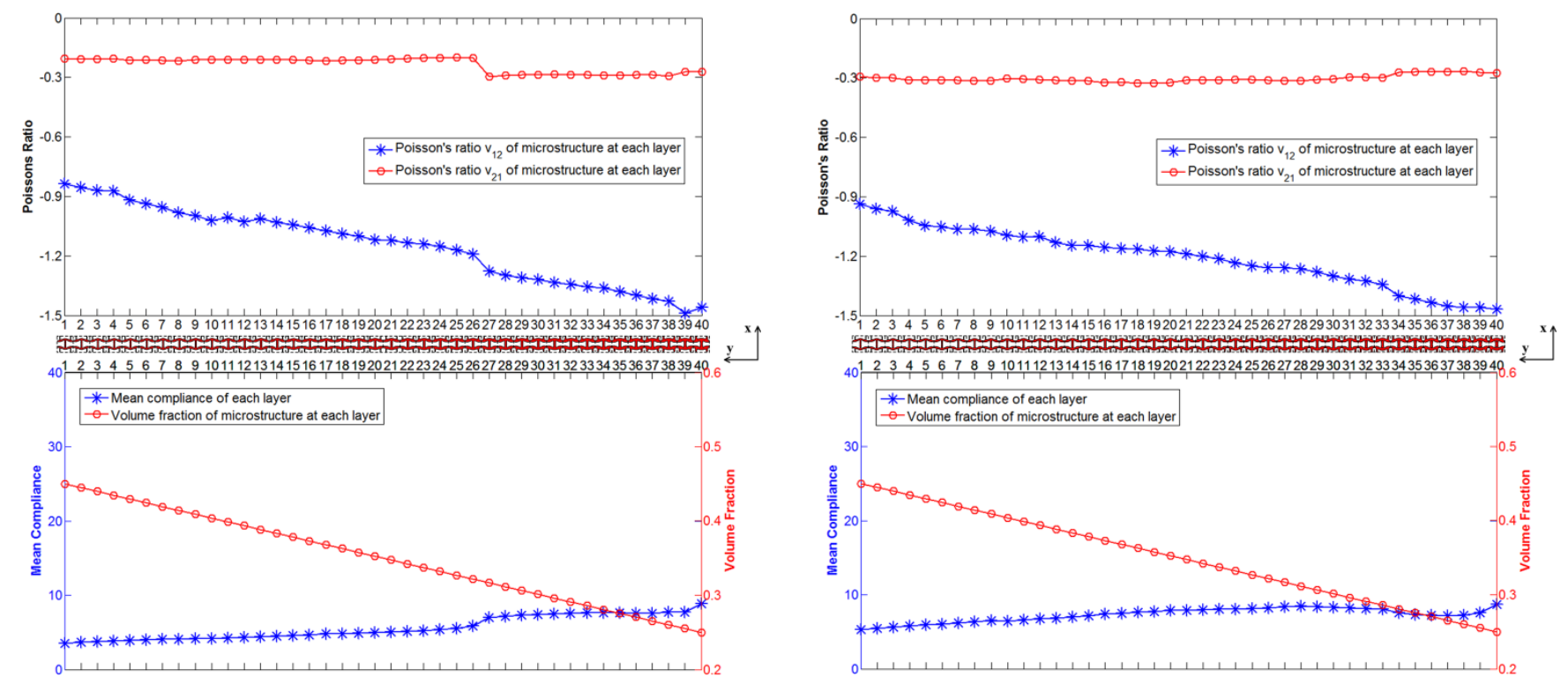

(c) Properties over 40 layers: Multi-objective design (Left); Single-objective design (Right)

Figure 17. Material properties with different layers

\section{Conclusions}

This paper has developed a level set-based topology optimization method for the computational design of functionally graded cellular composites hosting auxetic metamaterials. The multi-scale design problem is established as a multi-objective formulation to account for both structural stiffness and auxetic behavior of the material microstructure. PLSM is applied to evolve the design boundaries of the microstructures, with the evaluation of the effective properties of the microstructures achieved by using the numerical homogenization method. In numerical examples, a variety of topologically optimized auxetic composites with graded material properties have been achieved under specified loading and boundary conditions of the macrostructures. Numerical results show that the proposed method can be applied to design both isotropic and orthotropic material microstructures, to achieve desired properties. The optimized structure can satisfy multifunctional requirements with respect to practical applications of auxetic composites. Also all the topological designs show the favorable features of level set methods, as well as the benefits of the parametric level set method. Furthermore, based on the comparison of numerical cases, it can be found that the FGM composites with multi-layered microstructures perform better than the traditional designs only with identical microstructures. Actually, this method can be used to design any kind of new composites hosting periodic material microstructures fashioned as FGM layers. In engineering, the topologically 
optimized FGM composites with auxetics can be used to support a range of interesting applications, such as auxetic reinforced composites, crash energy absorption and etc.

\section{Acknowledgments}

This research is supported by National Basic Scientific Research Program of China (JCKY2016110C012), China Postdoctoral Science Foundation (2017M612446), Australian Research Council (ARC) - Discovery Projects (160102491), and National Natural-Science-Foundation of China (51575204).

\section{References}

[1] D.R. Smith, J.B. Pendry, M.C.K. Wiltshire, Metamaterials and negative refractive index, Science 305 (2004) 788-792.

[2] A. Sihvola, Metamaterials in electromagnetics, Metamaterials 1 (2007) 2-11.

[3] R. Lakes, Advances in negative Poisson's ratio materials, Adv. Mater. 5 (1993) 293-296.

[4] K.E. Evans, A. Alderson, Auxetic materials: functional materials and structures from lateral thinking, Adv. Mater. 12 (2000) 617-628.

[5] B. Xu, F. Arias, S.T. Brittain, X.M. Zhao, B. Grzybowski, S. Torquato, G.M. Whitesides, Making negative Poisson's ratio microstructures by soft lithography, Adv. Mater. 11 (1999) 1186-1189.

[6] R. Lakes, Negative Poisson's ratio materials, Science 238 (1987) 551.

[7] W. Yang, Z.M. Li, W. Shi, B. Xie, M. Yang, Review on auxetic materials, J. Mater. Sci. 39 (2004) 3269-3279.

[8] X.N. Hou, H. Hu, V. Silberschmidt, A novel concept to develop composite structures with isotropic negative Poisson's ratio: Effects of random inclusions, Compos. Sci. Technol. 72 (2012) 1848-1854.

[9] W. Zhang, Z.D. Ma, P. Hu, Mechanical properties of a cellular vehicle body structure with negative Poisson's ratio and enhanced strength, J. Reinf. Plast. Compos. 33 (2014) 342-349.

[10] L.J. Gibson, M.F. Ashby, Cellular Solids: Structure and Properties, 2nd Ed, Cambridge University Press, Cambridge, 1997.

[11] J.Y. Chen, Y. Huang, M. Ortiz, Fracture analysis of cellular materials: a strain gradient model, J. Mech. Phys. Solids 46 (1998) 789-828.

[12] R. Christensen, Mechanics of cellular and other low-density materials, Int. J. Solids Struct. 37 (2000) 93-104.

[13] J.M. Guedes, N. Kikuchi, Preprocessing and postprocessing for materials based on the homogenization method with adaptive finite element methods, Comput. Methods Appl. Mech. Engrg. 83 (1990) 143-198.

[14] O. Sigmund, Materials with prescribed constitutive parameters: An inverse homogenization problem, Int. J. Solids Struct. 31 (1994) 2313-2329.

[15] E. Andreassen, B.S. Lazarov, O. Sigmund, Design of manufacturable 3D extremal elastic microstructure, Mech. Mater. 69 (2014) 1-10.

[16] Y.Q. Wang, Z. Luo, N. Zhang, Z. Kang, Topological shape optimization of microstructural metamaterials using a level set method, Comput. Mater. Sci. 87 (2014) 178-186.

[17] Y. Miyamoto, W.A. Kaysser, B.H. Rabin, A. Kawasaki, R.G. Ford, Functionally Graded Materials: Design, Processing and Applications, Springer Science \& Business Media, Vol. 5, 2013. 
[18] E.C.N. Silva, M.C. Walters, G.H. Paulino, Modeling bamboo as a functionally graded material: lessons for the analysis of affordable materials, J. Mater. Sci. 41 (2006) 6991-7004.

[19] P. Coelho, P. Fernandes, H. Rodrigues, J. Cardoso, J. Guedes, Numerical modeling of bone tissue adaptation A hierarchical approach for bone apparent density and trabecular structure, J. Biomech. 42 (2009) 830-837.

[20] M. Koizumi, FGM activities in Japan, Compos. Pt. B-Eng. 28 (1997) 1-4.

[21] K. Ichikawa, Functionally graded materials in the 21st century, Kluwer, Boston, 2001.

[22] G.H. Paulino, E.C.N. Silva, C.H. Le, Optimal design of periodic functionally graded composites with prescribed properties, Struct. Multidiscip. Optim. 38 (2009) 469-489.

[23] V. Birman, L.W. Byrd, Modeling and analysis of functionally graded materials and structures, Appl. Mech. Rev. 60 (2007) 195-216.

[24] J.E. Cadman, S.W. Zhou, Y.H. Chen, Q. Li, On design of multi-functional microstructural materials, J. Mater. Sci. 48 (2013) 51-66.

[25] S.W. Zhou, Q. Li, Design of graded two-phase microstructures for tailored elasticity gradients, J. Mater. Sci. 43 (2008) 5157-5167.

[26] A. Radman, X.D. Huang, Y.M. Xie, Topology optimization of functionally graded cellular materials, J. Mater. Sci. 48 (2013) 1503-1510.

[27] W.H. Zhang, S.P. Sun, Scale-related topology optimization of cellular materials and structures, Int. J. Numer. Methods Eng. 68 (2006) 993-1011.

[28] J.D. Deng, J. Yan, G.D. Cheng, Multi-objective concurrent topology optimization of thermoelastic structures composed of homogeneous porous material, Struct. Multidiscip. Optim. 47 (2012) 583-597.

[29] X. Guo, X.F. Zhao, W.S. Zhang, J. Yan, G.M. Sun, Multi-scale robust design and optimization considering load uncertainties, Comput. Methods Appl. Mech. Engrg. 283 (2015) 994-1009.

[30] J. Yan, X. Guo, G.D. Cheng, Multi-scale concurrent material and structural design under mechanical and thermal loads, Comput. Mech. 57 (2016) 437-446.

[31] P.G. Coelho, H.C. Rodrigues, Hierarchical topology optimization addressing material design constraints and application to sandwich-type structures, Struct. Multidiscip. Optim. 52 (2015) 91-104.

[32] L. Xia, P. Breitkopf, Multiscale structural topology optimization with an approximate constitutive model for local material microstructure, Comput. Methods Appl. Mech. Engrg. 286 (2015) 147-167.

[33] M.P. Bendsøe, O. Sigmund, Topology Optimization: Theory, Methods, and Applications, Springer, Berlin, Heidelberg, 2003.

[34] M.P. Bendsøe, N. Kikuchi, Generating optimal topologies in structural design using a homogenization method, Comput. Methods Appl. Mech. Engrg. 71 (1988) 197-224.

[35] G. Allaire, E. Bonnetier, G. Francfort, F. Jouve, Shape optimization by the homogenization method, Numer. Math. 76 (1997) 27-68.

[36] G. Allaire, Shape optimization by the homogenization method, Applied Mathematical Sciences, vol. 146, Springer-Verlag, New York, 2002.

[37] M. Zhou, G.I.N. Rozvany, The COC algorithm, part II: Topological, geometry and generalized shape optimization, Comput. Methods Appl. Mech. Engrg. 89 (1991) 309-336.

[38] G.I.N. Rozvany, U. Kirsch, M.P. Bendsøe, O. Sigmund, Layout optimization of structures, Appl. Mech. Rev. 48 (1995) 41-119.

[39] Y.M. Xie, G.P. Steven, A simple evolutionary procedure for structural optimization, Comput. Struct. 49 (1993) 885-896. 
[40] S. Osher, J.A. Sethian, Fronts propagating with curvature-dependent speed-algorithms based on Hamilton-Jacobi formulations, J. Comput. Phys. 79 (1988) 12-49.

[41] J.A. Sethian, A. Wiegmann, Structural boundary design via level set and immersed interface methods, J. Comput. Phys. 163 (2000) 489-528.

[42] G. Allaire, F. Jouve, A.M. Toader, Structural optimization using sensitivity analysis and a level-set method, J. Comput. Phys. 194 (2004) 363-393.

[43] M.Y. Wang, X.M. Wang, D.M. Guo, A level set method for structural topology optimization, Comput. Methods Appl. Mech. Engrg. 192 (2003) 227-246.

[44] O. Sigmund, S. Torquato, Design of materials with extreme thermal expansion using a three-phase topology optimization method, J. Mech. Phys. Solids 45 (1997) 1037-1067.

[45] O. Sigmund, A new class of extremal composites, J Mech Phys Solids 48 (2000) 397-428.

[46] J.K. Guest, J.H. Prévost, Design of maximum permeability material structures, Comput. Methods Appl. Mech. Engrg. 196 (2007) 1006-1017.

[47] W.H. Zhang, G.M. Dai, F.W. Wang, S.P. Sun, H. Bassir, Using strain energy-based prediction of effective elastic properties in topology optimization of material microstructures, Acta Mech. Sin. 23 (2007) 77-89.

[48] A.R. Díaz, O. Sigmund, A topology optimization method for design of negative permeability metamaterials, Struct. Multidiscip. Optim. 41 (2009) 163-177.

[49] J. Schwerdtfeger, F. Wein, G. Leugering, R.F. Singer, C. Körner, M. Stingl, F. Schury, Design of auxetic structures via mathematical optimization, Adv. Mater. 23 (2011) 2650-2654.

[50] A. Radman, X.D. Huang, Y.M. Xie, Topological optimization for the design of microstructures of isotropic cellular materials, Eng. Optimiz. 45 (2013) 1331-1348.

[51] A. Faure, G. Michailidis, G. Parry, N. Vermaak, R. Estevez, Design of thermoelastic multi-material structures with graded interfaces using topology optimization, 2016. <hal-01383393>

[52] N.P. van Dijk, K. Maute, M. Langelaar, F. van Keulen, Level-set methods for structural topology optimization: a review, Struct. Multidiscip. Optim. 48 (2013) 437-472.

[53] A.L. Gain, G.H. Paulino, A critical comparative assessment of differential equation-driven methods for structural topology optimization, Struct. Multidiscip. Optim. 48 (2013) 685-710.

[54] T. Yamada, K. Izui, S. Nishiwaki, A. Takezawa, A topology optimization method based on the level set method incorporating a fictitious interface energy, Comput. Methods Appl. Mech. Engrg. 199 (2010) 2876-2891.

[55] P.D. Dunning, H.A. Kim, A new hole insertion method for level set based structural topology optimization, Int. J. Numer. Methods Eng. 93 (2013) 118-134.

[56] P.D. Dunning, H.A. Kim, Introducing the sequential linear programming level-set method for topology optimization, Struct. Multidiscip. Optim. 51 (2015) 631-643.

[57] X. Guo, W. Zhang, W. Zhong, Explicit feature control in structural topology optimization via level set method, Comput. Methods Appl. Mech. Engrg. 272 (2014) 354-378.

[58] Q. Xia, M.Y. Wang, T.L. Shi, A level set method for shape and topology optimization of both structure and support of continuum structures, Comput. Methods Appl. Mech. Engrg. 272 (2014) 340-353.

[59] K. Svanberg, The method of moving asymptotes: a new method for structural optimization, Int. J. Numer. Methods Eng. 24 (1987) 359-373.

[60] T. Belytschko, S.P. Xiao, C. Parimi, Topology optimization with implicitly function and regularization, Int. J. Numer. Methods Eng. 57 (2003) 1177-1196.

[61] Z. Luo, M.Y. Wang, S.Y. Wang, P. Wei, A level set-based parameterization method for structural shape and topology optimization, Int. J. Numer. Methods Eng. 76 (2008) 1-26. 
[62] Z. Luo, N. Zhang, W. Gao, H. Ma, Structural shape and topology optimization using a meshless Galerkin level set method, Int. J. Numer. Methods Eng. 90 (2012) 369-389.

[63] H. Li, P.G. Li, L. Gao, L. Zhang, T. Wu, A level set method for topological shape optimization of 3D structures with extrusion constraints, Comput. Methods Appl. Mech. Engrg. 83 (2015) 615-635.

[64] H. Wendland, Piecewise polynomial, positive definite and compactly supported radial functions of minimal degree, Adv. Comput. Math. 4 (1995) 389-396.

[65] T. Mori, K. Tanaka, Average stress in matrix and average elastic energy of materials with misfitting inclusions, Acta Metall. 21 (1973) 571-574.

[66] R. Hill, A self-consistent mechanics of composite materials, J. Mech. Phys. Solids 13 (1965) 213-222.

[67] R.T. Marler, J.S. Arora, Survey of multi-objective optimization methods for engineering, Struct. Multidiscip. Optim. 26 (2004) 369-395.

[68] H. Li, L. Gao, P.G. Li, Topology optimization of structures under multiple loading cases with a new compliancevolume product, Eng. Optimiz. 46 (2014) 725-744.

[69] K.K. Choi, N.H. Kim, Structural sensitivity analysis and optimization - Linear systems, Springer, NY, 2005.

[70] J. Sokolowski, J.P. Zolesio, Introduction to Shape Optimization: Shape Sensitivity Analysis, in: Springer Series in Computational Mathematics, vol. 10, Springer, New York, 1992.

[71] X.M. Wang, Y.L. Mei, M.Y. Wang, Level-set method for design of multi-phase elastic and thermoelastic materials, Int. J. Mech. Mater. Des. 1 (2004) 213-239. 